\title{
Green function, mean field equation and Painlevé VI equation
}

\author{
Chang-Shou Lin
}

\begin{abstract}
Recently jointly with C.L. Chai, Z.J. Chen, T.J. Kuo and C.L. Wang, we have developed a theory to connect different subjects such as (multiple) Green functions, mean field equations, Lamé equation, hyperelliptic curves, modular forms and Painlevé VI equation. Among others in those joint works, the premodular forms $Z_{r, s}^{(n)}(\tau)$ occupies the central role. We will discuss its role from the aspects of differential geometry (Green function), conformal geometry (mean field equations) and the classical integral Lamé equation. The key issue is to study the function properties of $Z_{r, s}^{(n)}(\tau)$ such as the simple zero properties and the asymptotics as $\tau \rightarrow+\infty$. Unexpectedly, Painlevé VI is the essential tool for the proof of these properties. The Okamoto transformation for Painlevé VI is used to set up the induction step. This is the key for many difficult computation. We also show how to apply the Painlevé properties to study the degenerate curves for branch points of the hyperelliptic curve $Y^{(n)}(\tau), n=2$.
\end{abstract}

\section{Contents}

1. Introduction

2. Green function and mean field equation 140

3. Hyper-elliptic curves 143

4. Modular forms 148

5. Generalized Lamé equations 154

6. Painlevé VI equation 164

7. A generalization of the Hitchin theorem 173

8. Application 182

$\begin{array}{ll}\text { References } & 187\end{array}$

\section{Introduction}

Throughout the paper, we fix the following notations $\omega_{0}=0, \omega_{1}=1$, $\omega_{2}=\tau, \omega_{3}=1+\tau, \wedge_{\tau}=\mathbb{Z}+\tau \mathbb{Z}$, and $E_{\tau} \doteqdot \mathbb{C} / \wedge_{\tau}$ where $\tau \in \mathbb{H}=\{\tau \mid \operatorname{Im} \tau>$ $0\}$. For a point $a \in \mathbb{C}$, we often denote it by $[a]$ to emphasize that this point 
$[a]$ is considered to be the point $a+\wedge_{\tau}$ in the torus $E_{\tau}$. We also define $E_{\tau}[2] \doteqdot\left\{\frac{\omega_{i}}{2} \mid i=0,1,2,3\right\}$ to be the set of the lattice point and 2-torsion points in $E_{\tau}$.

We recall $\wp(z \mid \tau), \zeta(z \mid \tau)$ and $\sigma(z)$ to be defined by:

$$
\begin{gathered}
\wp(z \mid \tau)=\frac{1}{z^{2}}+\sum_{\omega \in \wedge_{\tau} \backslash\{0\}}\left(\frac{1}{(z-\omega)^{2}}-\frac{1}{\omega^{2}}\right), \\
\zeta^{\prime}(z \mid \tau)=-\wp, \text { and } \zeta(z)=\frac{\sigma^{\prime}(z)}{\sigma(z)} .
\end{gathered}
$$

Here $\zeta(z \mid \tau)$ is odd and quasi-periodic:

$$
\zeta\left(z+\omega_{i} \mid \tau\right)=\zeta(z)+\eta_{i}(\tau), \quad i=1,2,3 .
$$

We refer [1] for the introduction to Weierstrass elliptic functions. Let $G(z \mid \tau)$ be the Green function on $E_{\tau}$ :

$$
\left\{\begin{array}{l}
\Delta G(z)=\delta_{0}-\frac{1}{\left|E_{\tau}\right|} \\
\int G(z) \mathrm{d} x \mathrm{~d} y=0
\end{array}\right.
$$

where $\delta_{0}$ is the Dirac measure at 0 and $\left|E_{\tau}\right|$ is the area of $E_{\tau}$.

In this article, we want to study critical points of $G^{(n)}(z \mid \tau)$ on $E_{\tau}^{n}=$ $E_{\tau} \times \cdots \times E_{\tau}$, where

$$
G^{(n)}(z \mid \tau)=n \sum_{i=1}^{n} G\left(z_{i}\right)-\sum_{i<j} G\left(z_{i}-z_{j}\right),
$$

under the deformation of $\tau$.

There are two types of critical points. A critical point $\left(a_{1}, \cdots, a_{n}\right)$ is called trivial if $\left\{a_{1}, \cdots, a_{n}\right\}=\left\{-a_{1}, \cdots,-a_{n}\right\}$, otherwise, it is called nontrivial. For each $n \in \mathbb{N}, G^{(n)}$ has $(2 n+1)$ trivial critical points. Locally each trivial critical point depends on $\tau$ holomorphically. A trivial critical point $a$ is called degenerate if the Hessian of $G^{(n)}(z)$ at $z=a$ is zero. We call the set of $\tau$ where trivial critical points $a(\tau)$ are degenerate as the degenerate curve in $\mathbb{H}$. The fundamental issue is whether the degenerate curve is smooth or not. This problem seems not trivial at all. It is unexpected that we could answer this questions by applying the Painlevé property of some Painlevé VI equation.

Indeed the study of critical points are related to mean field equations, Lamé equations, hyper-elliptic curves, modular forms and Painlevé VI equation. This is the purpose of the article to survey the links with these subjects.

In section 2 , we discuss the connection with the mean field equations. A critical point $a=\left(a_{1}, \cdots, a_{n}\right)$ of $G^{(n)}$ is related to the blowup set of a sequence of blowing up solutions of mean field equations. Indeed, our motivation for this project has been originated from studying the mean field equation at critical parameters. The mean field equation considered in section 2 is also an integrable system by the Liouville theorem. From this 
point of view, the mean field equation is related to the Lamé equation. See section 5 .

The set of critical points for $n=1$ are completely understand. See [27] and [10]. However even for $n=2$, the number of non-trivial critical points is still unsettled. In section 2 , we will discuss the cases $n=1$ and $n=2$ for this issue.

In section 3, we consider the classical Lamé equation and the hyperelliptic curve $Y_{n}(\tau)$ which is embedded in $E_{\tau}^{n}, n$ copies of $E_{\tau}$. The structure of the closure of $Y_{n}(\tau)$ has been thoroughly studied in [6] and [27]. Via the embedding into $E_{\tau}^{n}$, we naturally introduce the addition map $\sigma_{n}$ from $\overline{Y_{n}}(\tau)$ to $E_{\tau}$. In [27], the formula of degree $\sigma_{n}=\frac{n(n+1)}{2}$ has been obtained. This implies the function field $K\left(\overline{Y_{n}}(\tau)\right)$ is a finite extension of $K\left(E_{\tau}\right)$ and

$$
\left[K\left(\overline{Y_{n}}(\tau)\right): K\left(E_{\tau}\right)\right]=\frac{n(n+1)}{2} .
$$

The primitive element of this extension is denoted by $z_{n}(a)$ and we prove

$$
z_{n}(a)=\zeta\left(\sum_{i=1}^{n} a_{i}\right)-\sum_{i=1}^{n} \zeta\left(a_{i}\right) .
$$

Therefore, there is a minimal polynomial $W_{n}(X)$ such that $W_{n}\left(z_{n}(a)\right) \equiv 0$ in $\overline{Y_{n}}(\tau)$. Set

$$
Z_{r, s}^{(n)}(\tau)=W_{n}\left(Z_{r, s}(\tau)\right)
$$

where $Z_{r, s}(\tau)=\zeta(r+s \tau \mid \tau)-r \eta_{1}(\tau)-s \eta_{2}(\tau)$. We note that the pair $(r, s)$ is the monodromy data of the Lamé equation.

In section 4 , we see that $Z_{r, s}^{(n)}(\tau)$ is modular form of weight $\frac{n(n+1)}{2}$ w.r.t $\Gamma(N)$ provided that $(r, s)$ is a $N$-torsion point. The question:

Is every zero of $Z_{r, s}^{(n)}(\tau)$ simple?

had arisen in [27]. Interestingly, this question is answered affirmatively in [9]. The proof is to apply the Painlevé property of some Painlevé equation.

In section 6 , we briefly overview the basic theory of Painlevé VI equation and its elliptic form. We refer the readers to [23] for the excellent introduction of Painlevé VI equations. Two important features of Painlevé VI are: Painlevé property and the isomonodromic deformation. Clearly, Painlevé property could be applied only if the solutions has explicit expressions. In literature, the Hitchin theorem is the first result in this direction (except for the Picard solutions). In $\S 6$, we give two proofs of this remarkable result: one uses the isomodromic deformation, the other one is to apply the Okamoto transformation. By the Hitchin theorem, we immediately see how the algebraic solutions related to the Hecke form $Z_{r, s}(\tau)$. The Hecke form $Z_{r, s}(\tau)$ is a modular form if $(r, s)$ is $N$-torsion point. Hecke proved that $Z_{r, s}(\tau)$ is the Eisenstein series of weight 1 up to the constant $N$.

The Green function $G(z \mid \tau)$ has either three or five critical points. This was proved in [28]. Indeed $G\left(z \mid \tau_{0}\right)$ has five critical points iff there exists a real pair $(r, s) \in \mathbb{R}^{2} \backslash \frac{1}{2} Z^{2}$ such that $Z_{r, s}\left(\tau_{0}\right)=0$ holds. Denote this set of $\tau$ 
$\bmod \mathrm{SL}_{2}(z)$ by $\Omega^{(5)}$, and the interior of its complimentary set mod $\mathrm{SL}_{2}(Z)$ by $\Omega^{(3)}$. In $[\mathbf{1 0}]$, we prove both $\Omega^{(3)}$ and $\Omega^{(5)}$ are simply connected. In $\S 6$, we give a survey of this result.

This simple zero property of $Z_{r, s}^{(n)}(\tau)$ can be proved by applying the Painlevé VI equation. To do it, we need a generalization of Hitchin theorem. This is the main subject discussed in $\S 7$. In $\S 7$, we also discuss the explicit expression of non-completely reducible solutions for $n=2$. Form it, we prove the smoothness of degenerate curve for the trivial critical points of $G^{(2)}(z \mid \tau)$. So, we have seen that both completely reducible solutions or noncompletely reducible solution (the so-called Riccati type solutions) are useful for studying critical points of $G^{(n)}(z \mid \tau)$.

In the last section, we consider

$$
M_{N}^{(n)}(\tau)=\prod_{(r, s) \in Q_{N}} Z_{r, s}^{(n)}(\tau) .
$$

We calculate the vanishing order $\nu_{\infty}\left(M_{N}^{(n)}\right)$ of $M_{N}^{(n)}(\tau)$ at $\tau=\infty$ by using the Okamoto transformation. The function $M_{N}^{(n)}(\tau)$ is a modular form of weight $\frac{n(n+1)\left|Q_{N}\right|}{2}$ of level 1. The Okamoto transformation provides an induction process on $n$. Using the formula of $\nu_{\infty}\left(M_{N}^{(n)}\right)$, we solve the conjecture proposed by Dahmen and Beukers for the number of Lamé equations, under scalar equivalence, whose monodromy group is the dihedral group $D_{N}$ of order $2 N$.

\section{Green function and mean field equation}

2.1. Critical points of multiple Green function $E_{\tau}^{N}$. My original motivation is to understand the following problem: Consider $a=$ $\left(a_{1}, \cdots, a_{N}\right) \in E_{\tau} \times \cdots \times E_{\tau}$ satisfies

$$
\sum_{\ell=0}^{3} n_{\ell} \nabla G\left(a_{i}-\frac{\omega_{\ell}}{2}\right)=\sum_{j \neq i}^{N} \nabla G\left(a_{i}-a_{j}\right), \quad 1 \leq i \leq N,
$$

where $n_{\ell} \in \mathbb{N} \cup\{0\}$ and $N=\sum_{\ell=0}^{3} n_{\ell}$. Obviously, $a$ can be considered as a critical point of the Green function $G^{(\vec{n})}(z)$ on $E_{\tau}^{N}$ :

$$
G^{(\vec{n})}(z)=\sum_{i=1}^{N} \sum_{\ell=0}^{3} n_{\ell} G\left(z_{i}-\frac{\omega_{\ell}}{2}\right)-\sum_{i<j}^{N} G\left(z_{i}-z_{j}\right),
$$

where $\vec{n}=\left(n_{0}, n_{1}, n_{2}, n_{3}\right)$ and $z=\left(z_{1}, \ldots, z_{n}\right) \in E_{\tau}^{N}$.

Equation (2.1) is often related to the bubbling phenomena of a nonlinear elliptic PDE. For example, we consider

$$
\Delta u+e^{u}=8 \pi \sum_{i=0}^{3} n_{i} \delta\left(\frac{\omega_{i}}{2}\right) \text { in } E_{\tau},
$$


where $n_{i} \in \mathbb{N} \cup\{0\}, \delta(p)$ is the Dirac measure at $p$. We call $u$ is a solution of (3.1) if $u \in C^{\infty}\left(E_{\tau} \backslash E_{\tau}[2]\right)$ satisfies

$$
\Delta u+e^{u}=0 \text { in } E_{\tau} \backslash E_{\tau}[2]
$$

and

$$
u(z)=-2 n_{i} \log |z-q|+O(1) \text { as } z \rightarrow q \in E_{\tau}[2] .
$$

In conformal geometry, a solution $u$ of (1.2) is equivalent to saying that the Gaussian curvature of the new metric $\frac{1}{2} e^{u}|d z|^{2}$ is equal to 1 , but it has conic singularity at $\frac{\omega_{i}}{2}$. In this paper, we always assume $n_{i}$ are positive integers.

Suppose $u_{k}$ is a sequence of solutions of (3.1). A point $a \in E_{\tau}$ is called a blowup point if there are $x_{k}$ such that $x_{k} \rightarrow a$ and $u_{k}\left(x_{k}\right) \rightarrow+\infty$. Now assume $u_{k}$ blows up at $a_{1}, \cdots, a_{m}$. Assume $a_{i} \notin E_{\tau}[2]$. Then we have the following result from PDE:

TheOREM 2.1. $[\mathbf{1 1}, \mathbf{1 2}, \mathbf{1 3}]$ Suppose $\left\{a_{1}, \ldots, a_{m}\right\}$ is the blowup set of a sequence of solutions of (2.2) and $a_{i} \notin E_{\tau}[2]$. Then

(i) $m=N=\sum_{i=0}^{3} n_{i}$, and

(ii) $a_{1}, \cdots, a_{N}$ satisfies the balance condition (2.1).

The fact (i) is due to the quantization property of the entire solution of (2.2), a non-trivial result from PDE theory and the balance condition (2.1) is derived from the well-known Pohozaev identity.

2.2. Non-trivial critical points of Green function on $E_{\tau}$. Let $n=(1,0,0,0)$. Then $N=1$ and $(2.1)$ is reduced to

$$
\nabla G(a)=0,
$$

i.e., $a$ is a critical point of the Green function $G(z)$ on $E_{\tau}$. The Green function $G(z)$ is even, i.e., $G(-z)=G(z)$, and has singularity only at 0 , the lattice point. Thus, $G$ has at least three trivial critical points at half periods $\frac{\omega_{1}}{2}, \frac{\omega_{2}}{2}$ and $\frac{\omega_{3}}{2}$.

A critical point $a$ of $G$ is said non-trivial if $a \notin E_{\tau}[2]$. The corresponding mean field equation is

$$
\Delta u+e^{u}=8 \pi \delta_{0} \quad \text { in } E_{\tau} .
$$

Then we have the following connection of (2.3) and (2.4):

THEOREM 2.2. [28] The Green function $G(z)$ of $E_{\tau}$ has a non-trivial critical point iff (2.4) has a solution.

Naturally, we ask: How many non-trivial critical points at $E_{\tau}$ ? This question was answered in [28]:

THEOREM 2.3. [28] For each $\tau, G$ has at most one pair of non-trivial critical points $\pm a$. 
In [28], Theorem 2.3 was proved by using Alexander-Bol's inequality and the isoparametrical inequality. Later, Bergweiler and Eremenko [3] gave a proof by the dynamic arguments. If $\tau=i b, b>0$, then $G$ has no non-trivial critical points and then (2.4) has no solutions. But for $\tau=e^{i \pi / 3}, G$ has only one pair of non-trivial critical points $\pm \frac{\omega_{3}}{3}$ and (2.4) has an unique solution.

We remark that the proof of Theorem 2.3 is a consequence of the following PDE result:

For any $\tau \in \mathbb{H},(2.4)$ has at most one even solution.

This uniqueness for (2.4) is a highly non-trivial result. See [28]

In order to understand the set of critical points, one might deform $\tau$ starting from the imaginary axis. During this deformation process, we want to study the following questions:

(a) Where the bifurcation of critical point might occur?

(b) What is the geometry of those $\tau$ such that the Green function has nontrivial critical points.

The question (a) can be answered by Theorem 2.4.

THEOREM 2.4. The bifurcation of critical points of Green function could occurs only at trivial critical points (half periods).

REMARK 2.5. Suppose $G(z \mid \tau)$ has non-trivial critical points. Then $G\left(z \mid \tau^{\prime}\right), \tau^{\prime}=\gamma \cdot \tau, \gamma \in \mathrm{SL}(2, \mathbb{Z})$, also has non-trivial critical points. This is due to that fact $E_{\tau}$ and $E_{\tau^{\prime}}$ are holomorphically equivalent.

Let $\Omega_{5}=\{\tau \in \mathbb{H} \mid G(z \mid \tau)$ has non-trivial critical points $\}$. By the remark, $\Omega_{5}$ is invariant by the action of $\operatorname{SL}(2, \mathbb{Z})$. For some reason from the analytic point of view, we consider $\tau \in F_{2}$ :

$$
F_{2}=\left\{\tau \in \mathbb{H}|0 \leq \operatorname{Re} \tau \leq 1,| \tau-\frac{1}{2} \mid \geq \frac{1}{2}\right\}
$$

It is not difficult to see that $F_{2}$ is the closure of a fundamental domain of $\Gamma_{0}(2)$, where

$$
\Gamma_{0}(2)=\left\{\gamma \in \mathrm{SL}(2, \mathbb{Z}) \mid \gamma=\left(\begin{array}{ll}
a & b \\
c & d
\end{array}\right), c \equiv 0 \quad \bmod 2\right\} .
$$

For the simplicity, the set $\Omega_{5} \cap F_{2}$ is still denoted by $\Omega_{5}$ if there are no confusions. Similarly, we denote $\Omega_{3}$ by

$$
\Omega_{3}=\left\{\tau \in F_{2} \mid G(z \mid \tau) \text { has no non-trivial critical points }\right\} .
$$

Then we have the following theorem to describe the geometry of $\Omega_{5}$ and $\Omega_{3}$.

THEOREM 2.6. [10]

(i) $\Omega_{5}$ is open and simply connected.

(ii) $\partial \Omega_{5}=\partial \Omega_{3}$ consists of three smooth curves $C_{i}, i=1$, 2, and 3, where $C_{i}=\left\{\tau \in F_{2} \mid \frac{\omega_{i}}{2}\right.$ is a degenerate critical point of $\left.G(z \mid \tau)\right\}$.

(iii) $C_{i} \cap C_{j}=\emptyset$ 
We remark that (iii) implies that for any torus the Green function has at most one degenerate half period. This seems new in literature.

The proof of Theorem 2.6 is interesting in itself, a combination of some PDE results together with a simple fact of theory of modular form with respect to $\mathrm{SL}(2, \mathbb{Z})$ :

Any modular form of weight 8 has only one zero at $\tau=e^{\frac{i \pi}{3}}$.

The complete proof of Theorem 2.6 was given in [10]. In section 4, we will give a sketch of the proof after the discussion of connection of our problems with modular forms.

Naturally, for the general case we ask the similar questions to understand the solution structure of (2.1) when $\tau$ deforms in $\mathbb{H}$. Understandably, both questions (a) and (b) would be not easy to answer, because equation (2.1) is not an algebraic equation and the uniqueness of solutions of

$$
\Delta u+e^{u}=8 \pi n \delta_{0} \text { in } E_{\tau}
$$

no longer holds true for $n \geqslant 2$. Even through we still conjecture that Theorem 2.4 should be true for $n \geqslant 2$. But this is a very difficult question.

For question (b), we could say more than (a). Indeed, we will apply the Painlevé VI equations to prove the degenerate curves (in $\tau$ ) of trivial critical points are smooth. In section 7 , we will use $n=2$ as an example to explain how to apply the Painlevé VI equation for our purpose.

Equation (2.1) can be decomposed into two parts: an algebraic part and an non-algebraic part (one single equation). The equation for the algebraic part will give a hyper-elliptic structure, and it turns out this hyper-elliptic curve is identical with the spectral curve for the Treibich-Verdier potential in $\mathrm{KdV}$ theory. Let us to denote the hyper-elliptic curve by $Y^{(\vec{n})}$ and $X^{(\vec{n})}=$ $Y^{(\vec{n})} \backslash\{$ branch points $\}$. So, (2.1) is reduced to a single equation defined on $Y^{(\vec{n})}$. Like the case $n=(1,0,0,0)$, all the branch points of $Y^{(\vec{n})}$ are solutions to this single equation. See the Remark 3.3. We call $a=\left(a_{1}, \ldots, a_{N}\right) \in Y^{(\vec{n})}$ is a nontrivial solution of (2.1) if $a$ is not a branch point.

Geometrically, this single non-algebraic equation yields the global monodromy data for some linear ODE. For the multiple Green function, this ODE is the Lamé equation, which is the main subject discussed in section 5 .

\section{Hyper-elliptic curves}

3.1. The Green function and $Y^{(n)}(\tau)$. In this section, we want to introduce the hyper-elliptic curve $Y^{(n)}(\tau)$ associated with the multiple Green function through (2.1) and its embeddings into $E_{\tau}^{n}$. In this case, (2.1) becomes

$$
n \nabla G\left(a_{i}\right)=\sum_{j \neq i}^{n} \nabla G\left(a_{i}-a_{j}\right), 1 \leq i \leq N=n
$$


Clearly, any solution $a=\left(a_{1}, \ldots, a_{n}\right)$ of $(3.1)$ is a critical point of $G^{(n)}(z \mid \tau)$, where

$$
G^{(n)}(z \mid \tau)=n \sum_{i=1}^{n} G\left(z_{i} \mid \tau\right)-\sum_{i<j}^{n} G\left(z_{i}-z_{j} \mid \tau\right)
$$

for $z=\left(z_{1}, \ldots, z_{n}\right) \in E_{\tau}^{n}$.

First we recall $\zeta(z \mid \tau)=-\int \wp(z \mid \tau) \mathrm{d} z$ and $\eta_{i}(\tau), i=1,2$, are the quasiperiods. The Green function can be written in terms of Weierstrass elliptic functions, see (3.7) and (3.8) below. In particular,

$$
\frac{\partial G(z \mid \tau)}{\partial z}=\zeta(z \mid \tau)-\eta(z \mid \tau)
$$

where $\eta(z)$ is defined: if $z=r+s \tau$ for $r, s \in \mathbb{R}$, then

$$
\eta(r+s \tau)=r \eta_{1}(\tau)+s \eta_{2}(\tau) \text {. }
$$

By using (3.2), (3.1) can be rewritten as:

Proposition $3.1([\mathbf{6}])$. Let $a \in E_{\tau}^{n}$. Then $a=\left(a_{1}, \cdots, a_{n}\right)$ satisfies (3.1) if and only if

$$
\begin{gathered}
\sum_{j \neq i}^{n}\left(\zeta\left(a_{i}-a_{j}\right)+\zeta\left(a_{j}\right)-\zeta\left(a_{i}\right)\right)=0,1 \leq i \leq n, \text { and } \\
\sum_{i=1}^{n} \nabla G\left(a_{i}\right)=0 .
\end{gathered}
$$

The equivalence of (3.1) and (3.3)-(3.4) are straightforward computations. See [6]. The first equation, equation (3.3), is an algebraic equation by the addition theorem. Let us define $Y^{(n)}(\tau)$ is the quotient of the solution set of (3.3) by the permutation $S_{n}$ :

$\left\{a \in E_{\tau}^{n} \mid a_{i} \neq 0 \forall i\right.$ and $a_{i} \neq a_{j} \forall i \neq j$ such that (3.3) holds $\} / S_{n}$.

Then in [6] we could prove

THEOREM 3.2. [6]

(i) $Y^{(n)}(\tau)$ is a hyper-elliptic curve, i.e., there exists $\ell_{n}(B) \in$ $Q\left[g_{2}, g_{3}, B\right]$ of degree $2 n+1$ such that $C^{2}=\ell_{n}(B)$ holds, where

$$
C=\wp^{\prime}\left(a_{i}\right) \Pi_{j \neq i}\left(\wp\left(a_{i}\right)-\wp\left(a_{j}\right)\right) \text { and } B=(2 n-1) \sum_{i=1}^{n} \wp\left(a_{i}\right) \text {. }
$$

(ii) Except for a finite set of $\tau, Y^{(n)}(\tau)$ is smooth.

(iii) The branch points are the set of $\left(a_{1}, \cdots, a_{n}\right)$ such that $\left\{a_{1}, \cdots\right.$, $\left.a_{n}\right\}=\left\{-a_{1}, \cdots,-a_{n}\right\}$.

(iv) Let $a=\left(a_{1}, \ldots, a_{n}\right) \in Y^{(n)}(\tau)$. Then $a$ is not branch point iff $\left\{a_{1}, \cdots, a_{n}\right\} \cap\left\{-a_{1}, \cdots,-a_{n}\right\}=\emptyset$.

(v) Let $\overline{Y^{(n)}}(\tau)$ be the closure in $E_{\tau}^{n}$. Then $\overline{Y^{(n)}}(\tau)=Y^{(n)}(\tau) \cup$ $\{(0, \cdots, 0)\}$. 
(vi) $\overline{Y^{(n)}}(\tau)$ is the spectral curve of the potential $I_{0}(z)=n(n+1) \wp(z)$ in the $K d V$ theory.

REMARK 3.3. By (iii), any component $a_{i}$ of a branch point $a=$ $\left(a_{1}, \cdots, a_{n}\right)$ satisfies either $a_{i} \in E_{\tau}[2]$ (then $\left.\nabla G\left(a_{i}\right)=0\right)$ or the pair $\pm a_{i}$ appears in $a$ (then $\left.\nabla G\left(a_{i}\right)+\nabla G\left(-a_{i}\right)=0\right)$. Therefore, any branch point $a$ satisfies (3.4), i.e., any branch point is a solution to (3.1). By (i), there are $2 n+1$ distinct branch (finite) points if $Y^{n}(\tau)$ is smooth. Thus, $a=\left(a_{1}, \ldots, a_{n}\right)$ is called a non-trivial solution of (3.1) if $a$ is not a branch point of $Y^{(n)}(\tau)$. By (iv), we have

$$
\left\{a_{1}, \ldots, a_{n}\right\} \cap\left\{-a_{1},-a_{2}, \ldots,-a_{n}\right\}=\emptyset
$$

if $a$ is a non-trivial solution.

3.2. The branch points of $Y^{(2)}(\tau)$. Now we give some examples to compute these branch points. If $n=1, Y^{(n)}(\tau)=E_{\tau}$, and the branch points are the three half periods. For $n=2$, there are only five branch points which can be computed as follows:

Proposition 3.4. Suppose $n=2$. Then for each $\tau \in \mathbb{H}$, there are only five branch points of $Y^{(2)}(\tau)$ :

$$
\left(\frac{\omega_{i}}{2}, \frac{\omega_{j}}{2}\right) \quad 1 \leq i \neq j \leq 3 \text {, and }\left(q_{ \pm},-q_{ \pm}\right) \text {, }
$$

where $q_{ \pm}$satisfies $\wp\left(q_{ \pm}\right)= \pm \sqrt{\frac{g_{2}(\tau)}{12}}$.

Proof. Since the three half periods $\frac{1}{2} \omega_{i}, i=1,2,3$, are critical points of $G(z)$ and for any $i \neq j, \frac{1}{2} \omega_{i}-\frac{1}{2} \omega_{j}$ is the other half period, we see that $(a, b)=\left(\frac{1}{2} \omega_{i}, \frac{1}{2} \omega_{j}\right), i \neq j, i, j \in\{1,2,3\}$ satisfies (3.1). We can also find a solution $(a, b)$ for (3.1) satisfying $a=-b$ and $a$ is not a half period (i.e., $\left.\wp^{\prime}(a) \neq 0\right)$. In fact, since $\nabla G(z)$ is odd, (3.1) is equivalent to

$$
\nabla G(a)+\nabla G(b)=0, \quad \nabla G(a)-\nabla G(b)-\nabla G(a-b)=0 .
$$

On the other hand, the Green function $G$ could be expressed in terms of well-known functions:

$$
G(z)=-\frac{1}{2 \pi}\left(\ln \left|\vartheta_{1}(z)\right|-\frac{\pi}{\operatorname{Im} \tau}(\operatorname{Im} z)^{2}\right)+C(\tau)
$$

where

$$
\vartheta_{1}(z ; \tau) \doteqdot-i \sum_{n=-\infty}^{\infty}(-1)^{n} e^{\left(n+\frac{1}{2}\right)^{2} \pi i \tau} e^{(2 n+1) \pi i z}
$$

is the odd theta function and $C(\tau)$ is a constant; see [6]. From (3.7), we have

$$
\begin{aligned}
-4 \pi G_{z}(z) & =\zeta(z \mid \tau)-\eta_{1}(\tau) z+\frac{2 \pi i \operatorname{Im} z}{\operatorname{Im} \tau} \\
& =\zeta(z \mid \tau)-r \eta_{1}(\tau)-s \eta_{2}(\tau),
\end{aligned}
$$

where $z=r+s \tau, r, s \in \mathbb{R}$. In view of (3.8), the second equation in (3.6) becomes 


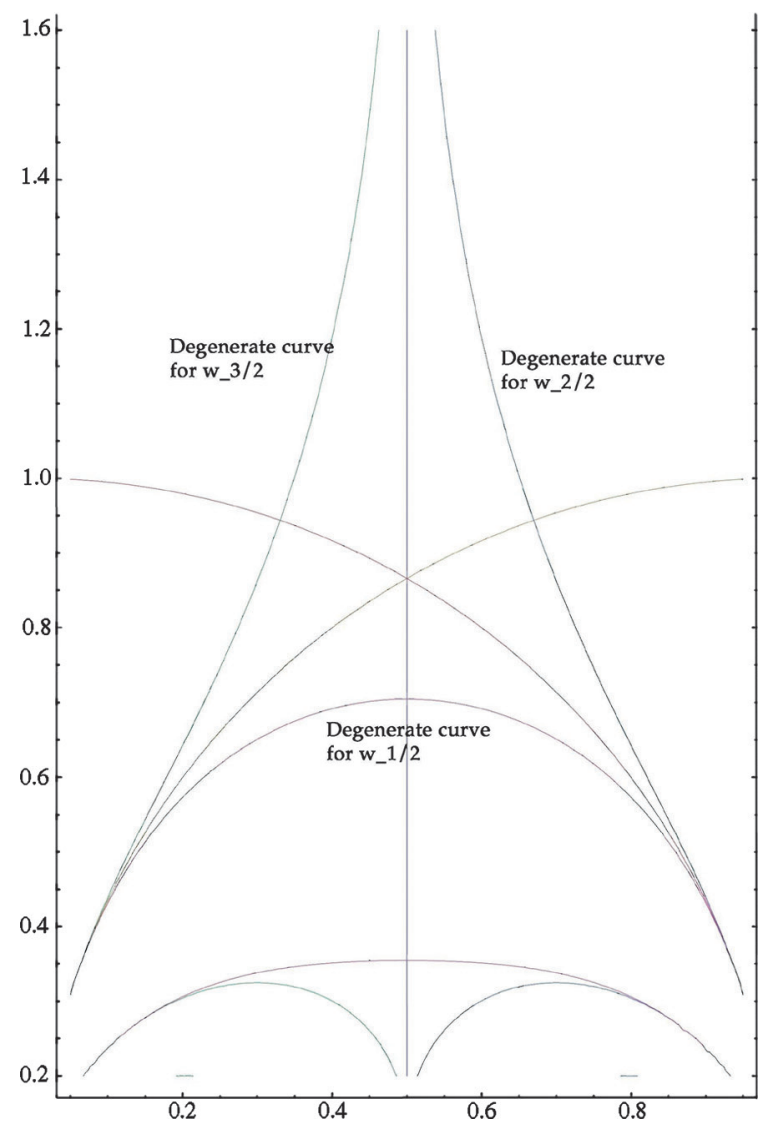

Figure 1.

$$
\zeta(a)-\zeta(b)-\zeta(a-b)=0 .
$$

If $a=-b$, then the first equation in (3.6) holds automatically, while (3.9) becomes $2 \zeta(a)-\zeta(2 a)=0$. This together with $\zeta(2 a)=2 \zeta(a)+\frac{1}{2} \frac{\wp^{\prime \prime}(a)}{\wp^{\prime}(a)}$, implies that $a$ satisfies $\wp^{\prime \prime}(a)=0$. Since $\wp^{\prime \prime}(z)=6 \wp^{2}(z)-\frac{g_{2}}{2}$, a satisfies

$$
\wp(a)=\wp(-a)= \pm \sqrt{\frac{g_{2}}{12}} .
$$

REMARK 3.5. This proves $\wp\left(q_{ \pm}\right)= \pm \sqrt{\frac{g_{2}}{12}}$ iff $\wp^{\prime \prime}\left(q_{ \pm}\right)=0$.

Let $F_{2}$ be the fundamental domain of $\Gamma_{0}(2)$ :

$$
F_{2}=\left\{\tau|0 \leq \operatorname{Re} \tau \leq 1,| \tau-\frac{1}{2} \mid \geq \frac{1}{2}\right\},
$$

For $n=1$, let $C_{i}$ be the curve for those $\tau$ such that $\frac{\omega_{i}}{2}$ is a degenerate critical point of $G(z \mid \tau)$. Figure 1 is the numerical simulation of those degenerate curves, which is confirmed by Theorem 2.6. 


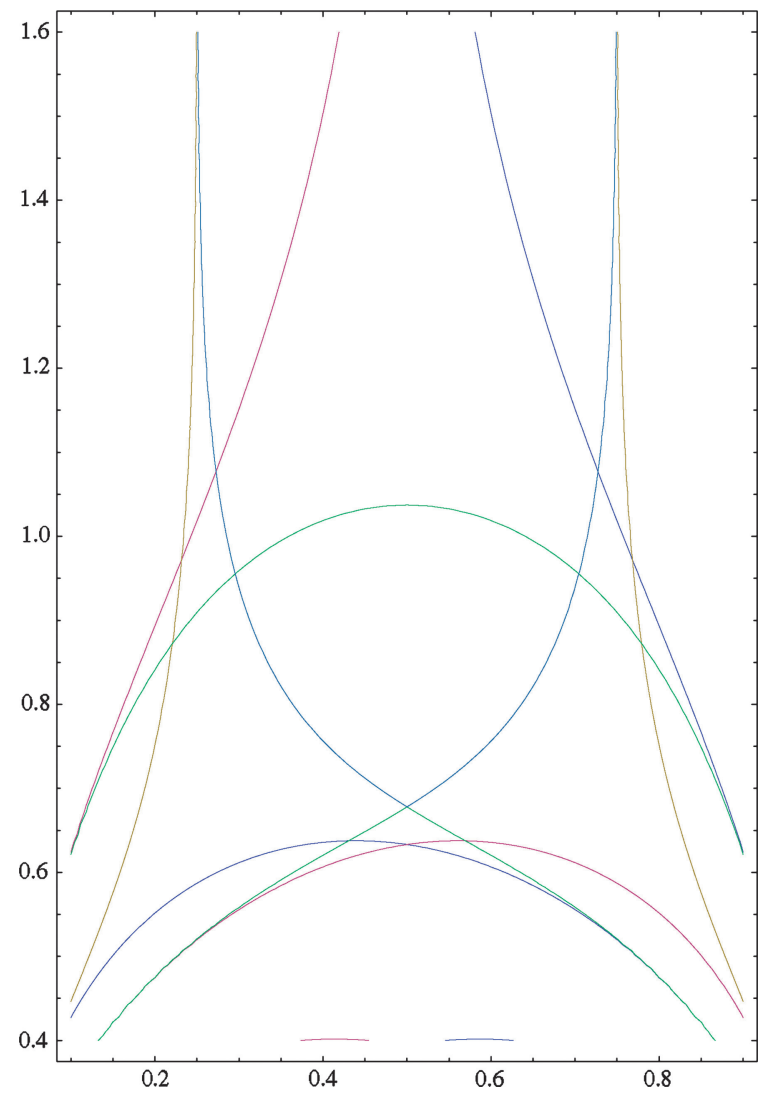

FiguRE 2 .

For $n=2$, we let $C_{i j}$ be the degenerate curve for $\left(\frac{\omega_{i}}{2}, \frac{\omega_{j}}{2}\right)$, i.e., $\left(\frac{\omega_{i}}{2}, \frac{\omega_{j}}{2}\right)$ is a degenerate critical point of the Green $G^{(2)}$ function on $E_{\tau} \times E_{\tau}$. Similarly, $C_{ \pm}$is the degenerate curve for $\left(q_{ \pm}(\tau),-q_{ \pm}(\tau)\right)$.

Theorem 3.6. For $\tau \in F$, all the degenerate curves $C_{i j}$ and $C_{ \pm}$are smooth.

By computer simulation, those curves are shown in Figure 2. The fundamental domain $F_{2}$ is divided into several regions by $C_{i j}$ and $C_{ \pm}$, and in each region, the number of non-trivial solution to (3.1) should be the same. However it is still an open problem because this problem is related to the question (a) in section 2. So far, we could prove it only if the following conjecture holds true:

Except of a finite set of $\tau$, the bifurcation of critical points could occur only at branch points of $Y^{(n)}(\tau)$

Theorem 3.6 is an application of the Painlevé VI equation (Riccati equation). A sketch of the proof of Theorem 3.6 is given in section 7. See Theorem 7.6. 


\section{Modular forms}

4.1. Hecke forms. Recall the derivative of $G$ : write $z=r+s \tau, r, s \in \mathbb{R}$

$$
-4 \pi G_{z}(z)=\zeta(r+s \tau)-r \eta_{1}(\tau)-s \eta_{2}(\tau) .
$$

Now fix $(r, s) \in \mathbb{C} \times \mathbb{C}$, we denote the RHS of the above by $Z_{r, s}(\tau)$ :

$$
Z_{r, s}(\tau)=\zeta(r+s \tau)-r \eta_{1}(\tau)-s \eta_{2}(\tau)
$$

Some properties of $Z_{r, s}$ :

Proposition 4.1. (i) If $(r, s) \in \mathbb{R}^{2} \backslash\{(0,0)\}$, then $Z_{r, s}(\tau)$ is a holomorphic function in $\tau \in \mathbb{H}$.

(ii) If $(r, s) \in\left\{\left( \pm \frac{1}{2}, \pm \frac{1}{2}\right)\right\} \bmod 1$, then $Z_{r, s}(\tau) \equiv 0$ in $\tau$.

(iii) If $(r, s) \in \mathbb{C} \times \mathbb{C} \backslash \mathbb{R} \times \mathbb{R}$, then $Z_{r, s}(\tau)$ has a simple pole at $\tau_{0}$ such that $r+s \tau_{0} \in \wedge_{\tau_{0}}$, where $\wedge_{\tau_{0}}$ is the lattice generated by 1 and $\tau_{0}$.

(iv) $Z_{r, s}(\tau)$ satisfies the following modularity:

$$
Z_{r^{\prime}, s^{\prime}}\left(\tau^{\prime}\right)=(c \tau+d) Z_{r, s}(\tau)
$$

$$
\text { if } \gamma=\left(\begin{array}{ll}
a & b \\
c & d
\end{array}\right) \in \mathrm{SL}(2, \mathbb{Z}),(r, s)=\left(r^{\prime}, s^{\prime}\right) \cdot \gamma \text {, and } \tau^{\prime}=\gamma \cdot \tau \doteqdot \frac{a \tau+b}{c \tau+d} \text {. }
$$

Hence if $(r, s)=\left(\frac{k_{1}}{N}, \frac{k_{2}}{N}\right)$ is a $N$-torsion point $\left(\operatorname{gcd}\left(k_{1}, k_{2}, N\right)=1\right)$, then by (4.2) $Z_{r, s}(\tau)$ is a modular form of weight one w.r.t. $\Gamma_{N}$. This was first proved by Hecke [21] on 1926. Furthermore, Hecke noted that $Z_{r, s}(\tau)$ is the Eisenstein series of weight 1 . We recall that the Eisenstein series of weight 1 is defined by

$$
\mathfrak{E}_{1}^{N}\left(\tau, s ; k_{1}, k_{2}\right):=(\operatorname{Im} \tau)^{s} \sum_{(m, n)}(m \tau+n)^{-n}|(m \tau+n)|^{-2 s},
$$

where $(m, n)$ runs over $\mathbb{Z}^{2}$ under the condition $0 \neq(m, n) \equiv\left(k_{1}, k_{2}\right) \bmod N$. It is known that $\mathfrak{E}_{1}^{N}\left(\tau, s ; k_{1}, k_{2}\right)$ is a meromorphic function in the $s$-plane and holomorphic at $s=0$. Set $\mathfrak{E}_{1}^{N}\left(\tau ; k_{1}, k_{2}\right):=\mathfrak{E}_{1}^{N}\left(\tau, 0 ; k_{1}, k_{2}\right)$. It can be proved (see $[\mathbf{1 7}])$ that if $(r, s)$ is a $N$-torsion point, then

$$
Z_{r, s}(\tau)=N \mathfrak{E}_{1}^{N}\left(\tau ; k_{1}, k_{2}\right) \text { if }(r, s) \equiv\left(\frac{k_{1}}{N}, \frac{k_{2}}{N}\right) \quad \bmod \mathbb{Z} .
$$

Recall $F_{2}$ is the fundamental domain of $\Gamma_{0}(2)$. The following result was proved in $[\mathbf{1 0}]$.

THEOREM 4.2. [10]

(i) For any pair $(r, s) \notin \mathbb{C}^{2} \backslash \frac{1}{2} Z^{2}, Z_{r, s}(\tau)=\zeta(r+\zeta \tau)-\left(r \eta_{1}(\tau)+s \eta_{2}(\tau)\right)$ has simple zeros only.

(ii) Suppose $(r, s) \in \mathbb{R}^{2} \backslash\left\{\left( \pm \frac{1}{2}, \pm \frac{1}{2}\right)\right\}$. Then $Z_{r, s}(\tau)$ has a zero at $\tau \in F_{2}$ iff $\pm(r, s) \in \Delta_{2} \bmod 1$, where

$$
\Delta_{2}=\left\{(r, s) \mid 0 \leq r \leq \frac{1}{2}, 0 \leq s \leq \frac{1}{2}, 0 \leq r+s \leq 1\right\} .
$$

Moreover, the zero is unique in $F_{2}$. 
REMARK 4.3. Since $Z_{m \pm r, n \pm s}(\tau)= \pm Z_{r, s}(\tau)$ for integers $m, n$, for any real pair $(r, s)$ we might assume $(r, s) \in \square=\left\{(r, s) \mid 0 \leq r \leq 1,0 \leq s \leq \frac{1}{2}\right\}$. Then we decompose the rectangle $\square$ as the union of four triangles as follows. Thus Theorem 4.2 (ii) says that $Z_{r, s}(\tau)$ has exactly one zero if $(r, s) \in \Delta_{2}$,

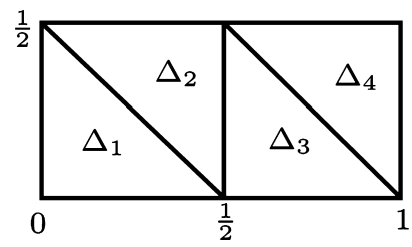

and has no zeros if $(r, s) \in \Delta_{1} \cup \Delta_{3} \cup \Delta_{4} \cup_{i=1}^{4} \partial \Delta_{i}$. Let $\tau=\tau(r, s)$ be the unique zero of $Z_{r, s}(\tau)$ in $F_{2}$. Then $\tau(r, s)$ is a 1-1 map from $\Delta_{2}$ onto $\Omega_{5}$, a consequence of Theorem 2.3. Since $\Delta_{2}$ is simply connected, we have $\Omega_{5}$ is simply connected.

PRoOF. We will prove (ii) and (i) for the case $(r, s) \in \mathbb{R}^{2}$. The general case will be proved in section 6 .

To prove (ii), we should use the PDE result and the theory of modular form. The PDE result is about the non-existence of the following nonlinear equation

$$
\Delta u+e^{u}=8 \pi \delta(0) \text { in } E_{\tau}
$$

if $E_{\tau}$ is a rectangle i.e. $\tau=i b, b>0$, where $\delta(0)$ is the Dirac measure at $0 \in E_{\tau}$. See [28]. A function $u \in C^{\infty}\left(E_{\tau} \backslash\{0\}\right)$ is said a solution to (4.3) if $\Delta u+e^{u}=0$ in $E_{\tau} \backslash\{0\}$ and $u$ satisfies

$$
u(z)=4 \log |z|+O(1) \text { as } z \rightarrow 0 .
$$

This non-existence of (4.3) for $\operatorname{Re} \tau=0$ implies that for any $(r, s) \in \mathbb{R}^{2} \backslash \frac{1}{2} Z^{2}$,

$$
Z_{r, s}(\tau) \neq 0 \quad \forall \tau=i b, b>0 .
$$

Since $E_{\tau^{\prime}} \approx E_{\tau}$ if $\tau^{\prime}=\gamma \cdot \tau,(4.4)$ implies

$$
Z_{r, s}(\tau) \neq 0, \text { for any } \tau \in \partial F \cap \mathbb{H} \text {. }
$$

The second ingredient is to apply the theory of the modular forms with respect to $\mathrm{SL}(2, \mathbb{Z})$. It is easy to see that the product $\prod_{(r, s): 3 \text {-torsion }} Z_{r, s}(\tau)$ is a modular form of weight 8 with respect to $\operatorname{SL}(2, \mathbb{Z})$. There are only one modular form of weight 8 up to constants. This implies that $Z_{\frac{1}{3}, \frac{1}{3}}(\tau)$ has only zero at $\tau=\rho=e^{\frac{\pi i}{3}}$, and the zero is a simple zero.

The following lemma shows that if $(r, s)$ is a $N$-torsion point with $N=4$ or 6 , then $Z_{r, s}(\tau) \neq 0 \forall \tau \in \mathbb{H}$.

LEMMA 4.4. For any $\tau \in \mathbb{H}$,

(i) $\zeta\left(\frac{3}{4} \omega_{1}+\frac{1}{4} \omega_{2}\right) \neq \frac{3}{4} \eta_{1}+\frac{1}{4} \eta_{2}$.

(ii) $\zeta\left(\frac{1}{6} \omega_{1}+\frac{1}{6} \omega_{2}\right) \neq \frac{1}{6} \eta_{1}+\frac{1}{6} \eta_{2}$. 
(iii) $\zeta\left(\frac{2}{6} \omega_{1}+\frac{3}{6} \omega_{2}\right) \neq \frac{2}{6} \eta_{1}+\frac{3}{6} \eta_{2}$.

The proof of Lemma 4.4 is a good exercise of the addition theorem. Now we can prove (ii) of Theorem 4.2 .

Step 1: we compute the asymptotic of $Z_{r, s}(\tau)$ as $\tau$ tends to three cusp points $\infty, 0,1$ :

$$
\begin{aligned}
& \lim _{\tau \rightarrow \infty} Z_{r, s}(\tau)= \begin{cases}2 \pi i\left(s-\frac{1}{2}\right) & \text { if } s \neq 0, \\
\pi \cot \pi r & \text { if } s=0,\end{cases} \\
& \lim _{\tau \rightarrow 0} Z_{r, s}(\tau)= \begin{cases}\frac{2 \pi i}{\tau}\left(\frac{1}{2}-r\right) & \text { if } r \in(0,1), \\
\frac{\pi \cot \pi s}{\tau} & \text { if } r=0,\end{cases} \\
& \lim _{\tau \rightarrow 1} Z_{r, s}(\tau)= \begin{cases}\frac{2 \pi i}{\tau-1}\left(\frac{1}{2}-(r+s)\right) & \text { if } r+s \in(0,1), \\
\frac{\pi \cot \pi s}{\tau-1} & \text { if } r+s=0 .\end{cases}
\end{aligned}
$$

For the details of computation, we refer [10]. In particular if $(r, s) \notin$ $\left\{\left(r, \frac{1}{2}\right) \mid 0 \leq r<1\right\} \cup\left\{\left(\frac{1}{2}, s\right) \mid 0 \leq s<\frac{1}{2}\right\} \cup\left\{(r, s) \mid r+s=\frac{1}{2}\right\}$, then the limit of $Z_{r, s}(\tau)$ does not vanish as $\tau$ tends to any cusp $\in\{0,1, \infty\}$.

Step 2: By Lemma 4.4, $Z_{r, s}(\tau)$ has no zero at $\left(\frac{3}{4}, \frac{1}{4}\right),\left(\frac{1}{6}, \frac{1}{6}\right)$ and $\left(\frac{2}{6}, \frac{3}{6}\right)$ which are contained in $\Delta_{1}$ and $\Delta_{3} \cup \Delta_{4} \cup\{(r, s) \mid r+s=1\}$. Since $\lim _{\tau \rightarrow \partial F} Z_{r, s}(\tau) \neq 0$ $\forall(r, s) \in \Delta_{1} \cup \Delta_{3} \cup \Delta_{4} \cup\left\{(r, s) \mid r+s=\frac{1}{2}\right\}$ by Step 1 and the PDE result, we conclude that $Z_{r, s}(\tau)$ has no zeros in $F$ for $(r, s) \in \Delta_{1} \cup \Delta_{3} \cup \Delta_{4} \cup\{(r, s) \mid r+s=$ $\left.\frac{1}{2}\right\}$. Consequently, for $(r, s) \in \bigcup_{i=1}^{4} \partial \Delta_{i}, Z_{r, s}(\tau)$ has no solutions.

For $(r, s) \in \Delta_{2}$, we have the number of zeros of $Z_{r, s}(\tau)=$ the number of zeros of $Z_{\frac{1}{3}, \frac{1}{3}}(\tau)$. Then (ii) and (i) for real pairs $(r, s)$ follows easily.

REMARK 4.5. The advantage of our method that $Z_{r, s}(\tau)$ for $(r, s) \notin Q^{2}$ is used to connect one modular form (with a $N_{1}$-torsion point $(r, s)$ ) with another modular form (with a $N_{2}$-torsion point). During the deformation, the zeros are contained within the interior of $F_{2}$.

4.2. To construct premodular forms. Next we will construct the modular form for $n \geq 2$. For the family of hyper-elliptic curves $Y^{(n)}(\tau)$, $\tau \in \mathbb{H}$, we could associate a "premodular form" $Z_{r, s}^{(n)}(\tau)$. Here $Z_{r, s}^{(n)}(\tau)$ is called a premodular form if $Z_{r, s}^{(n)}(\tau)$ is a modular form w.r.t $\Gamma(N)$ for any $N$-torsion point $(r, s)$.

ThEOREM 4.6. [27] For any pair $(r, s) \in \mathbb{R}^{2} \backslash \frac{1}{2} \mathbb{Z}^{2}$, there exists a premodular form $Z_{r, s}^{(n)}(\tau)$ of weight $\frac{n(n+1)}{2}$ satisfying the following property:

$$
\begin{aligned}
& Z_{r_{0}, s_{0}}^{(n)}\left(\tau_{0}\right)=0 \text { for some }\left(r_{0}, s_{0}\right) \notin \frac{1}{2} \mathbb{Z}^{2} \text { iff there } i s \text { an } \\
& a=\left(a_{1}, \cdots, a_{n}\right) \in Y^{(n)}\left(\tau_{0}\right) \text { satisfying (3.4) and } \sum_{i=1}^{n} a_{i} \notin E_{\tau_{0}}[2]
\end{aligned}
$$


or equivalently, there exists a non-trivial solution a to (3.1) such that $\sum_{i=1}^{n} a_{i} \notin$ $E_{\tau_{0}}[2]$.

We will give a proof of Theorem 4.6 later after some necessary preparations. To construct $Z_{r, s}^{(n)}(\tau)$, we consider two maps $\sigma_{n}$ and $z_{n}$ on $Y^{(n)}(\tau)$ :

(i) $\sigma_{n}(a)=\sum_{i=1}^{n} a_{i}$ is the addition map from $Y^{(n)}(\tau)$ to $E_{\tau}$ and

(ii) $z_{n}(a)=\zeta\left(\sum_{i=1}^{n} a_{i}\right)-\sum_{i=1}^{n} \zeta\left(a_{i}\right)$ is a meromorphic function from $\overline{Y^{(n)}}(\tau)$ to $\mathbb{P}^{1}=\mathbb{C} \cup\{\infty\}$.

Recall $\overline{Y^{(n)}}=Y^{(n)} \cup\{(0, \ldots, 0)\}$. It implies that $z_{n}(a)$ has a (simple) pole at those $a$ such that either $a=(0,0, \ldots, 0)$ or $\sum a_{i}=0 \bmod \wedge_{\tau}$. How many $a=\left(a_{1}, \ldots, a_{n}\right)$ with $\sum a_{i}=0 \bmod \wedge_{\tau}$ ? The answer is given by the following result.

TheOREM 4.7. [27] Both $\sigma_{n}$ and $z_{n}$ have the degree $\frac{n(n+1)}{2}$.

Theorem 4.7 is the main result in [27]. The part on $\sigma_{n}$ is proved by applying the cubics theorem in algebraic geometry. The degree formula implies that if $\sigma_{0} \in E_{\tau}$ is not a critical value, then $\sigma_{n}^{-1}\left(\sigma_{0}\right)$ has $\frac{n(n+1)}{2}$ element. Through $\sigma_{n}$, the function field $K\left(\overline{Y^{(n)}}(\tau)\right)$ of $\overline{Y^{(n)}}(\tau)$ is an extension of $K\left(E_{\tau}\right)$ with

$$
\left[K\left(\overline{Y^{(n)}}(\tau)\right): K\left(E_{\tau}\right)\right]=\frac{n(n+1)}{2} .
$$

So we want to know what is the primitive element of this extension. It turns out $z_{n}$ is the primitive element. In fact we could prove there exists a monic polynomial $W_{n}(X ; \sigma) \in Q\left[g_{2}, g_{3}, \wp(\sigma), \wp^{\prime}(\sigma), X\right]$ where $g_{2}$ and $g_{3}$ are coefficients of $E_{\tau}: y^{2}=4 x^{3}-g_{2} x-g_{3}$ such that

(iii) $\operatorname{deg} W=\frac{n(n+1)}{2}$,

(iv) $W_{n}\left(z_{n}(a) ; \sigma_{n}(a)\right) \equiv 0$ in $Y^{(n)}(\tau)$,

(v) $W_{n}$ is homogeneous in $g_{2}, g_{3}, \wp(\sigma), \wp^{\prime}(\sigma)$ and $X$ if we assign them the weights by $4,6,2,3,1$ respectively.

The fact that $z_{n}$ is a primitive element is equivalent to the part of Theorem 4.7 concerning the degree of $z_{n}$, as a map from $\overline{Y^{(n)}}$ to $\mathbb{P}^{1}$. It is equivalent to prove that the values of $z_{n}(a)$ are distinct for $a \in \sigma_{n}^{-1}\left(\sigma_{0}\right)$ for generic $\sigma_{0}$, i.e.,

(4.6) if $\sigma_{n}(a)=\sigma_{n}\left(a^{\prime}\right)$ and $z_{n}(a)=z_{n}\left(a^{\prime}\right)$ for $a, a^{\prime} \in Y^{(n)}(\tau)$ then $a=a^{\prime}$.

The precise statement about (4.6) is the following lemma. See [27].

LEMMA 4.8. [27] Suppose a is not a branch point of $Y^{(n)}(\tau)$ and $\sigma_{n}(a)=$ $\sigma_{n}\left(a^{\prime}\right)$ for two distinct points $a, a^{\prime} \in Y^{(n)}(\tau)$. If $z_{n}(a)=z_{n}\left(a^{\prime}\right)$ then $a=a^{\prime}$. In particular, the preimage $\sigma_{n}^{-1}(r+s \tau)$ consists of $\frac{n(n+1)}{2}$ distinct elements in $Y^{(n)}(\tau)$ provided that $(r, s) \in \mathbb{R}^{2} \backslash \frac{1}{2} \mathbb{Z}^{2}$. 
There are at least two ways to prove Lemma 4.8, one is our original proof in [27], which extend the classical method of the so-called second symmetric (tensor) product of the Lamé equation. Note that the classical second symmetric product is for solutions of the same equation, but our proof can be extended to solutions of different equations, but with the same type. Another method is to use the Painlevé VI, and Lemma 4.8 is a special case of a general theorem see $[\mathbf{9}]$.

Let us come back to our subject $W_{n}$. Once $W_{n}$ is known, we can construct our premodular form $Z_{r, s}^{(n)}(\tau)$ by

$$
Z_{r, s}^{(n)}(\tau)=W_{n}\left(Z_{r, s}(\tau) ; \sigma_{n}\right), \quad \sigma_{n}=r+s \tau,
$$

where $Z_{r, s}(\tau)$ is the Hecke form:

$$
Z_{r, s}(\tau)=\zeta(r+s \tau \mid \tau)-r \eta_{1}(\tau)-s \eta_{2}(\tau)
$$

We remark that $Z_{r, s}^{(n)}(\tau)$ could be defined for all $(r, s) \in \mathbb{C}^{2} \backslash \frac{1}{2} Z^{2}$.

Recall that $Z_{r, s}(\tau)$ is a modular form of weight 1 w.r.t $\Gamma(N)$ if $(r, s)$ is a $N$-torsion point. This together with (v) implies that $Z_{r, s}^{(n)}(\tau)$ is a modular form of weight $\frac{n(n+1)}{2}$ w.r.t $\Gamma(N)$.

Proof of TheOREM 4.6. Note that if $Z_{r_{0}, s_{0}}^{(n)}\left(\tau_{0}\right)=0$ and $\left(r_{0}, s_{0}\right) \notin \frac{1}{2} \mathbb{Z}^{2}$ then $Z_{r_{0}, s_{0}}\left(\tau_{0}\right)$ is a root of $W\left(X ; \sigma_{n}\right)$ and $\sigma_{n}=r_{0}+s_{0} \tau_{0}$. By Lemma 4.8, $W\left(X ; \sigma_{n}\right)$ has $\frac{n(n+1)}{2}$ distinct roots, which are $\left\{z_{n}(a) \mid a \in \sigma_{n}^{-1}\left(r_{0}+s_{0} \tau_{0}\right)\right\}$. Therefore there exist $a \in Y^{(n)}(\tau)$ such that

$$
r+s \tau=\sigma_{n}(a)=\sum_{i=1}^{n} a_{i}, \text { and } Z_{r, s}\left(\tau_{0}\right)=z_{n}(a) .
$$

Recall the definition:

$$
z_{n}(a)=\zeta\left(\sum a_{i}\right)-\sum_{i=1}^{n} \zeta\left(a_{i}\right)
$$

So, the second equation of (4.8) implies

$$
\sum_{i=1}^{n} \zeta\left(a_{i}\right)=r \eta_{1}+s \eta_{2}
$$

To link (4.9) with (3.4), we recall

$$
G_{z}\left(a_{i}\right)=\zeta\left(a_{i}\right)-\left(r_{i} \eta_{1}+s_{i} \eta_{2}\right), \quad a_{i}=r_{i}+s_{i} \tau
$$

Then (4.9) is identical with (3.4):

$$
\begin{aligned}
\sum_{i=1}^{n} G_{z}\left(a_{i}\right) & =\sum_{i=1}^{n} \zeta\left(a_{i}\right)-\left(\left(\sum r_{i}\right) \eta_{1}+\left(\sum s_{i}\right) \eta_{2}\right) \\
& =\sum_{i=1}^{n} \zeta\left(a_{i}\right)-r \eta_{1}-s \eta_{2}=0 .
\end{aligned}
$$


Therefore $a$ satisfies (3.4). This proves the sufficient part.

Conversely if there is $a=\left(a_{1}, \ldots, a_{n}\right) \in Y^{(n)}\left(\tau_{0}\right)$ satisfying (3.4), then

$$
0=\sum_{i=1}^{n} G_{z}\left(a_{i}\right)=\sum_{i=1}^{n} \zeta\left(a_{i}\right)-\left(\sum r_{i}\right) \eta_{1}-\left(\sum s_{i}\right) \eta_{2} .
$$

Since $r+s \tau_{0}=\sum_{i=1}^{n} a_{i}=\left(\sum r_{i}\right)+\left(\sum s_{i}\right) \tau_{0}$, we have $r=\sum r_{i}$ and $s=\sum s_{i}$, which implies $\sum_{i=1}^{n} \zeta\left(a_{i}\right)=r \eta_{1}+s \eta_{2}$, and $z_{n}(a)=\zeta\left(\sum a_{i}\right)-\sum_{i=1}^{n} \zeta\left(a_{i}\right)=$ $\zeta\left(r+s \tau_{0}\right)-\left(r \eta_{1}+s \eta_{2}\right)=Z_{r, s}\left(\tau_{0}\right)$. Therefore, $Z_{r, s}^{(n)}\left(\tau_{0}\right)=W\left(Z_{r, s}\left(\tau_{0}\right)\right)=$ $W\left(z_{n}(a), \sigma_{n}(a)\right)=0$.

REMARK 4.9. If a real pair $\left(r_{0}, s_{0}\right) \notin \frac{1}{2} Z^{2}$ and $Z_{r_{0}, s_{0}}^{(n)}\left(\tau_{0}\right)=0$ for some $\tau_{0}$, then by Theorem 4.6, $r_{0}+s_{0} \tau_{0}$ is a non-trivial critical point of $G^{n}\left(z \mid \tau_{0}\right)$. Since $Z_{r, s}(\tau)$ is holomorphic in $\tau$ by the Rouche theorem, the zero $\tau$ of $Z_{r, s}(\tau)$ is well-defined as a (real) analytic function of $(r, s)$. Thus $Z_{r, s}^{(n)}(\tau)$ has a zero near $\tau_{0}$ for any $(r, s)$ near $\left(r_{0}, s_{0}\right)$. The local behavior of $\tau(r, s)$ is related the question (a) in section 2. For example if we could prove $\tau(r, s)$ is an open mapping, then question (a) might be answered.

In $\S 5$, we see that the zeros of $Z_{r, s}^{(n)}(\tau)$ also consists of those tori such that (3.1) has non-trivial solutions, or equivalently, the mean field equation,

$$
\Delta u+e^{u}=8 \pi n \delta_{0} \quad \text { in } E_{\tau},
$$

has at least one solution. See Theorem 5.2.

The most important property of $Z_{r, s}^{(n)}(\tau)$, as a holomorphic function in $\mathbb{H}$, is the following simple zero property.

TheOREM 4.10. [9] For $(r, s) \in \mathbb{C}^{2} \backslash \frac{1}{2} \mathbb{Z}^{2}, Z_{r, s}^{(n)}(\tau)$ has simple zeros only.

Theorem 4.10 was conjectured in [27]. Recently, Chen-Kuo-Lin proves this highly non-trivial result by applying the Painlevé VI equations. We will briefly discuss the proof in $\S 7$.

We remark that the explicit expression of $W_{n}(X ; \sigma)$ is very difficult to compute even for small $n$. For $n=2$ and 3, it was obtained by Dahmen [15], who used the classical formula (the so-called Frobenius-Stickelberger formula) to obtain it and for $n=4$, it was obtained by Wang and the author [27] by applying the addition map $\sigma_{n}$ and the setting above. Here we list some known examples from $[\mathbf{2 7}]$ and $[\mathbf{1 5}]$.

- For $n=2$,

$$
\begin{aligned}
& W^{(2)}(X ; \sigma)=X^{3}-3 \wp(\sigma) X-\wp^{\prime}(\sigma), \text { and } \\
& Z_{r, s}^{(2)}(\tau)=Z_{r, s}^{(3)}(\tau)-3 \wp(\sigma) Z_{r, s}(\tau)-\wp^{\prime}(\sigma) .
\end{aligned}
$$

Obviously, $Z_{r, s}^{(2)}(\tau)$ is a modular form of weight 3 w.r.t. $\Gamma(N)$, if $(r, s)$ is a $N$-torsion point. 
- For $n=3, W^{(3)}(X ; \sigma)$ is a polynomial of degree 6 :

$$
\begin{aligned}
W^{(3)}(X ; \sigma)= & X^{6}-15 \wp(\sigma) X^{4}-20 \wp^{\prime}(\sigma) X^{3}+\left(\frac{27}{4} g_{2}-45 \wp^{2}(\sigma)\right) X^{2} \\
& -12 \wp(\sigma) \wp^{\prime}(\sigma) X-\frac{5}{4} \wp^{\prime}(\sigma)^{2}
\end{aligned}
$$

- For $n=4, W^{(4)}(X ; \sigma)$ is a polynomial of degree 10 :

$$
\begin{aligned}
W^{(4)}(X ; \sigma)= & X^{10}-45 \wp(\sigma) X^{8}-120 \wp^{\prime}(\sigma) X^{7}+\left(\frac{399}{4} g_{2}-630 \wp^{2}(\sigma)\right) X^{6} \\
& -504 \wp(\sigma) \wp^{\prime}(\sigma) X^{5}-\frac{15}{4}\left(280 \wp^{3}(\sigma)-49 g_{2} \wp(\sigma)-115 g_{3}\right) X^{4} \\
& +15\left(11 g_{2}-24 \wp^{2}(\sigma)\right) \wp^{\prime}(\sigma) X^{3} \\
& -\frac{9}{4}\left(140 \wp^{4}(\sigma)-245 g_{2} \wp^{2}(\sigma)+190 g_{3} \wp(\sigma)+21 g_{2}^{2}\right) X^{2} \\
& -\left(40 \wp^{3}(\sigma)-163 g_{2} \wp(\sigma)+125 g_{3}\right) \wp^{\prime}(\sigma) X \\
& +\frac{3}{4}\left(25 g_{3}-3 \wp^{2}(\sigma)\right) \wp^{\prime}(\sigma)^{2} .
\end{aligned}
$$

\section{Generalized Lamé equations}

5.1. Mean field equation and the Lamé equation. The classic Lamé equation is a Heun ordinary differential equation with four regular singular points (one of them is $\infty$ ):

$$
p(x) \frac{\mathrm{d}^{2} y}{\mathrm{~d} x^{2}}+\frac{1}{2} p^{\prime}(x) \frac{\mathrm{d} y}{\mathrm{~d} x}-(n(n+1) x+B) y=0, \quad x \in \mathbb{C}
$$

where $p(x)=4 x^{3}-g_{2}(\tau) x-g_{3}(\tau)$. By using the two-one projection $x=$ $\wp(z \mid \tau)$ from $E_{\tau}$ to $\mathbb{C} \cup\{\infty\},(5.1)$ is transferred to

$$
y^{\prime \prime}(z)=(n(n+1) \wp(z)+B) y(z), \quad z \in E_{\tau} .
$$

Classically, (5.1) is called the algebraic form of the Lamé equation, and (5.2) is called the elliptic form of (5.1). In this paper, the Lamé equation is often referred as the elliptic form. For (5.2), $n$ is called the index and $B$ is called the accessory parameter of the Lamé equation.

The Lamé equation has been thoroughly studied since 19th century, in particular the case $n \in \frac{1}{2} \mathbb{Z}$. When $n \in \frac{1}{2} \mathbb{Z}$, the monodromy representation is generally reduced to a homomorphism from $\pi_{1}\left(E_{\tau}\right)$ to $\operatorname{GL}(2, \mathbb{C})$. We refer the readers to $[\mathbf{9}]$ for the full discussion of the Lamé equation with $n \in \frac{1}{2} \mathbb{Z}$. For the rest of this subsection, we consider $n \in \mathbb{N}$, and (5.1) is called an integral Lamé equation.

From the geometric aspects, there is an important link between the Lamé equation and the mean field equation:

$$
\Delta u+e^{u}=8 \pi n \delta_{0} \quad \text { in } E_{\tau} .
$$


where $n \in \mathbb{N}$. Equation (5.3) is considered to be an integrable system in the following sense: for any solution $u(z)$ of (5.3), there exists an meromorphic function $f(z)$ on $\mathbb{C}$ such that

$$
u(z)=\log \frac{8\left|f^{\prime}(z)\right|^{2}}{\left(1+|f(z)|^{2}\right)^{2}}, \quad z \in E_{\tau} .
$$

This meromorphic function $f$ is called a developing map of $u$. For $n \in \mathbb{R}^{+}$, $f(z)$ is multi-valued. However if $n \in \frac{1}{2} \mathbb{Z}$ then $f(z)$ becomes a single-valued function. To recover $f(z)$, we note that $u_{z z}-\frac{1}{2} u_{z}^{2}$ is meromorphic because

$$
\left(u_{z z}-\frac{1}{2} u_{z}^{2}\right)_{\bar{z}}=4\left(u_{z} e^{u}-\left(e^{u}\right)_{z}\right)=0 .
$$

Since $u_{z z}-\frac{1}{2} u_{z}^{2}$ has singularities only at 0 , we have

$$
u_{z z}-\frac{1}{2} u_{z}^{2}=-2(n(n+1) \wp(z)+B)
$$

for some complex number B.

On the other hand, by the formula (5.4), we have

$$
u_{z z}-\frac{1}{2} u_{z}^{2}=\frac{f^{\prime \prime \prime}}{f^{\prime}}-\frac{3}{2}\left(\frac{f^{\prime \prime}}{f^{\prime}}\right)^{2} .
$$

Thus if $f_{1}$ and $f_{2}$ are two developing maps of $u$, then the classical Schwarz theorem says that

$$
f_{2}(z)=\frac{a f_{1}(z)+b}{c f_{2}(z)+d} \quad \text { for some } \gamma=\left(\begin{array}{ll}
a & b \\
c & d
\end{array}\right) \in \mathrm{SL}(2, \mathbb{C}) .
$$

Due to the restriction $(5.4)$, we have $\gamma \in \mathrm{SU}(2, \mathbb{C})$. Then the question is how to connect (5.1) and (5.3). We recall a classical result (See [35]).

Proposition 5.1. Suppose $y_{1}$ and $y_{2}$ are linearly independent solutions of (5.1), and set $f=\frac{y_{2}}{y_{1}}$. Then the Schwarz derivative

$$
\{f ; z\}=-2(n(n+1) \wp(z)+B) .
$$

Therefore, the developing map $f$ is a ratio of two independent solution of (5.1), where $B$ is determined by (5.5). From this connection, we see that the monodromy group for $f$ is the projective monodromy group of the corresponding Lamé equation.

On one hand, we could compute the monodromy group for $f$ by the geometry of the mean field equation. We note that $f(z)$ is not necessary to be doubly periodic. Indeed, after a suitable normalization, $f$ satisfies

$$
f(z+1)=e^{2 \pi i \theta_{1}} f(z) \text { and } f(z+\tau)=e^{2 \pi i \theta_{2}} f(z), \text { for } \theta_{i} \in \mathbb{R} .
$$

There is a simple, but important, observation. If $f$ is a developing map satisfying (5.6), then $f_{\lambda}(z)=e^{\lambda} f(z), \lambda \in \mathbb{R}$, also satisfies (5.6). Then plugging it into (5.4), the "rescaled" $u_{\lambda}$ defined by

$$
u_{\lambda}(z)=\log \frac{8 e^{2 \lambda}\left|f^{\prime}(z)\right|^{2}}{\left(1+e^{2 \lambda}|f(z)|^{2}\right)^{2}},
$$


is doubly periodic and satisfies the same mean field equation. Then we could easily see that as $\lambda \rightarrow \infty$, the blowup set of $u_{\lambda}$ consists of the zeros of $f$. Similarly, as $\lambda \rightarrow-\infty$, the blowup set of $u_{\lambda}$ consists of the poles of $f$. Clearly, by (5.6) the zeros or the poles of $f$ are well-defined as points in $E_{\tau}$. From the PDE result, any blowup set does not contain the origin 0 and has $n$ elements. By Theorem 2.1 (ii), both $a=\left(a_{1}, \ldots, a_{n}\right)$ and $b=\left(b_{1}, \ldots, b_{n}\right)$ satisfy (3.1) which implies $a, b \in Y^{(n)}(\tau)$ and both satisfy (3.4). Furthermore, we could prove $a=-b$. Clearly $\left\{a_{1}, \ldots, a_{n}\right\} \cap\left\{b_{1}, \ldots, b_{n}\right\}=\emptyset$. Thus $b=-a$ implies $\left\{a_{1}, \ldots, a_{n}\right\} \cap\left\{-a_{1}, \ldots,-a_{n}\right\}=\emptyset$, that is, $a$ is not a branch point of $Y^{(n)}(\tau)$ (see Theorem 3.2 (iv)). Let $\sigma_{n}(a)=r+s \tau$ for some $(r, s) \in \mathbb{R}^{2}$. In order to apply Theorem 4.6 , we need to prove $(r, s) \notin \frac{1}{2} \mathbb{Z}^{2}$. Indeed, it was proved in [6]. Now we can apply Theorem 4.6 in $\S 4$, which implies that $Z_{r, s}^{(n)}(\tau)=0$. This proves the sufficient part of the following result.

TheOREm 5.2. Let $\tau \in \mathbb{H}$. Then (5.3) has a solution iff there exists a real pair $(r, s) \notin \frac{1}{2} \mathbb{Z}^{2}$ such that $Z_{r, s}^{(n)}(\tau)=0$.

Proof. For the necessary part, we also use the sufficient part of Theorem 4.6: there exists $a=\left(a_{1}, \ldots, a_{n}\right) \in Y^{(n)}(\tau)$ such that $a$ satisfies (3.1) and $\sum_{i=1}^{n} a_{i}=r+s \tau$. Since $(r, s) \notin \frac{1}{2} \mathbb{Z}^{2}, a$ must be a non-branch point of $Y^{(n)}(\tau)$. Set

$$
f_{a}(z):=\prod_{i=1}^{n} \exp \int_{0}^{z} \frac{\wp^{\prime}\left(a_{i}\right)}{\wp(\omega)-\wp\left(a_{i}\right)} \mathrm{d} \omega .
$$

Then by a direct computation, we could prove $f_{a}(z)$ satisfies (5.6) for some real constant $\theta_{1}$ and $\theta_{2}$, and the function $u(z)$ defined by (5.4) satisfies the mean field equation. For a complete proof, we refer $\S 5$ and $\S 6$ of $[6]$.

5.2. The projective monodromy group of developing maps. Recall the monodromy group for $f$ is a homomorphism $\bar{\rho}$ from $\pi_{1}\left(E_{\tau}\right)$ to $\operatorname{PSL}(2, \mathbb{C})$. Hence the monodromy group is an Abelian group generated by $\bar{\rho}\left(\ell_{i}\right), i=1,2$, where $\ell_{i}$ is the fundamental cycles of $E_{\tau}$ represented by the loop $[0,1]$ and $[0, \tau]$ respectively. The identity (5.6) precisely says that

$$
\bar{\rho}\left(L_{1}\right)= \pm\left(\begin{array}{cc}
e^{\pi i \theta_{1}} & 0 \\
0 & e^{-\pi i \theta_{1}}
\end{array}\right) \text { and } \bar{\rho}\left(L_{2}\right)= \pm\left(\begin{array}{cc}
e^{\pi i \theta_{2}} & 0 \\
0 & e^{-\pi i \theta_{2}}
\end{array}\right) .
$$

We claim

$$
\theta_{1}=-2 s \text { and } \theta_{2}=2 r .
$$

The claim (5.8) can be proved by (5.7) as follows. We recall the addition formula:

$$
\frac{1}{2} \frac{\wp^{\prime}(z)}{\wp(z)-\wp(a)}=\zeta(z+a)-\zeta(z)-\zeta(a) .
$$


We apply (5.9) to (5.7), and have

$$
f(z)=e^{2 z \sum_{i=1}^{n} \zeta\left(a_{i}\right)} \frac{\prod_{i=1}^{n} \sigma\left(z-a_{i}\right)}{\prod_{i=1}^{n} \sigma\left(z+a_{i}\right)} .
$$

The transformation law

$$
\sigma\left(z+\omega_{i}\right)=-e^{\eta_{i}\left(z+\frac{\omega_{i}}{2}\right)} \sigma(z) .
$$

gives the transformation law for $f(z)$ :

$$
f\left(z+\omega_{i}\right)=f(z) \exp \left(2 \omega_{i} \sum_{j=1}^{n} \zeta\left(a_{j}\right)-2 \eta_{i} \sum_{j=1}^{n} a_{j}\right) .
$$

Thus

$$
\begin{aligned}
& \pi i \theta_{1}=\sum_{j=1}^{n} \zeta\left(a_{j}\right)-\eta_{1} \sum_{j=1}^{n} a_{j}=\left(\sum_{j=1}^{n} \zeta\left(a_{j}\right)-\left(\eta_{1} r+s \eta_{2}\right)\right)-2 \pi i s, \\
& \pi i \theta_{2}=\tau \sum_{j=1}^{n} \zeta\left(a_{j}\right)-\eta_{2}(r+s \tau)=\tau\left(\sum_{j=1}^{n} \zeta\left(a_{j}\right)-\left(\eta_{1} r+s \eta_{2}\right)\right)+2 \pi i r .
\end{aligned}
$$

But (3.4) implies

$$
\sum_{j=1}^{n} \zeta\left(a_{j}\right)=\eta_{1} r+\eta_{2} s \quad \text { if } \quad \sum a_{j}=r+s \tau, r, s \in \mathbb{R}
$$

Hence (5.8) follows. So if the Lamé equation comes from the mean field equation, then the projective monodromy group is generated by

$$
\pm\left(\begin{array}{cc}
e^{-2 \pi i s} & 0 \\
0 & e^{2 \pi i s}
\end{array}\right) \text { and } \pm\left(\begin{array}{cc}
e^{2 \pi i r} & 0 \\
0 & e^{-2 \pi i r}
\end{array}\right)
$$

where by $(5.12),(r, s)$ is determined by the zeros $\left\{z_{1}, \cdots, z_{n}\right\}$ of $f$.

5.3. Monodromy group of integral Lamé equation. Now we want to calculate the monodromy group for any Lamé equation (not necessarily related to mean field equation), (5.10) suggests that the fundamental solutions $y_{1}$ and $y_{2}$ should be given by

$$
\begin{aligned}
& y_{1}(z)=y_{a}(z) \doteqdot e^{z \sum_{i=1}^{n} \zeta\left(a_{i}\right)} \frac{\prod_{i=1}^{n} \sigma\left(z-a_{i}\right)}{\sigma(z)^{n}}, \text { and } \\
& y_{2}(z)=y_{-a}(z) \doteqdot e^{-z \sum_{i=1}^{n} \zeta\left(a_{i}\right)} \frac{\prod_{i=1}^{n} \sigma\left(z+a_{i}\right)}{\sigma(z)^{n}} .
\end{aligned}
$$

Indeed, this is the so-called Hermite-Halphen Ansatz [35] in the literature for any integral Lamé equation (5.2), which does not necessarily come from 
the mean field equation. By a straightforward computation, we have the following theorem.

TheOREm 5.3. [6] Let $a=\left(a_{1}, \ldots, a_{n}\right) \in E_{\tau}^{n}$. Then $y_{a}(z)$ satisfies (5.2) iff $a \in Y^{(n)}(\tau)$. Moreover,

$$
B=(2 n-1) \sum_{i=1}^{n} \wp\left(a_{i}\right)
$$

REMARK 5.4. If $a$ is not a branch point of $Y^{(n)}(\tau)$ then $y_{a}(z)$ and $y_{-a}(z)$ are two independent solutions of (5.2) with $B=(2 n-1) \sum_{i=1}^{n} \wp\left(a_{i}\right)$. Then the monodromy group for (5.2) is generated by

$$
\left(\begin{array}{cc}
e^{-2 \pi i s} & 0 \\
0 & e^{2 \pi i s}
\end{array}\right) \text { and }\left(\begin{array}{cc}
e^{2 \pi i r} & 0 \\
0 & e^{-2 \pi i r}
\end{array}\right),
$$

where $(r, s) \in \mathbb{C}^{2} \backslash \frac{1}{2} \mathbb{Z}^{2}$ satisfies

$$
\begin{aligned}
\sum_{i=1}^{n} a_{i} & =r+s \tau, \text { and } \\
\sum_{i=1}^{n} \zeta\left(a_{i}\right) & =r \eta_{1}+s \eta_{2} .
\end{aligned}
$$

since the matrix $\left(\begin{array}{cc}1 & \tau \\ \eta_{1} & \eta_{2}\end{array}\right)$ is invertible for any $\tau \in \mathbb{H},(r, s)$ can always be solved. Note that $\sum_{i=1}^{n} a_{i}$ and $\sum_{i=1}^{n} \zeta\left(a_{i}\right)$ ate related to maps $\sigma_{n}(a)$ and $z_{n}(a)$ in $\S 4$.

We have only given a quick overview about the integral Lamé equation and its connection with the mean field equation. For the complete theory including the generalized Lamé equations, we refer [6], [8] and [27].

5.4. Dahmen-Beukers conjecture. In this subsection we would like to discuss a conjecture proposed by Dahmen and Beukers. See $[\mathbf{4}, \mathbf{1 5}]$. Obviously, the Lamé equation possesses some symmetry from $S L(2, \mathbb{Z})$ action. For example, if we let $\tau^{\prime}=\gamma \cdot \tau:=\frac{a \tau+b}{c \tau+d}$ for some $\gamma=\left(\begin{array}{ll}a & b \\ c & d\end{array}\right) \in S L(2, \mathbb{Z})$ and make a scalar change of the independent variable $z \mapsto(c \tau+d) z$, then equation (5.2) is transformed to

$$
y^{\prime \prime}(z)=\left(n(n+1) \wp\left(z \mid \tau^{\prime}\right)+(c \tau+d)^{2} B\right) y(z) \text { in } E_{\tau^{\prime}} .
$$

These scalar changes of variable induce a natural equivalence relation on the space of all Lamé equations; we call these two Lamé equations (5.2) and (5.16) scalar equivalent. 
Equivalently, two Lamé equations of the algebraic form (5.1) are scalar equivalent if one can be transformed into the other by changing of variable $x \mapsto a x$ for some $a \in \mathbb{C} \backslash\{0\}$.

Conventionally, people are interested in (5.1) with algebraic solutions only, or equivalently with a finite monodromy group; see $[\mathbf{4}, \mathbf{1 5}, \mathbf{3 5}]$ and references therein. It is known that for an integral Lamé equation, if the monodromy group is finite, then the monodromy group $M$ of (5.1) is a dihedral group $D_{N}$ of order $2 N$ for some $N \in \mathbb{N}_{\geq 3}$. Let $L_{n}(N)$ denote the number of Lamé equations (5.1) or (5.2) modulo scalar equivalence with monodromy group dihedral $D_{N}$. It is well known (cf. $[4, \mathbf{1 5}, \mathbf{1 6}]$ ) that $L_{n}(N)$ is finite for given $n \in \mathbb{N}$ and $N \in \mathbb{N}_{\geq 3}$. The issue is how to compute it. In [15] Dahmen and Beukers proposed an explicit formula about $L_{n}(N)$; see (5.22) below.

Denote the number of Lamé equations (5.1) modulo scalar equivalence with projective monodromy group dihedral $D_{N}$ by $P L_{n}(N)$. The well-known Klein theorem asserts that any second order Fuchsian differential equation on $\mathbb{C P}^{1}$ has finite projective monodromy group if and only if it is a pull-back of a hypergeometric equation belonging to the basic Schwarz list. Therefore, every integral Lamé equation (5.1) with projective monodromy group dihedral $D_{N}$ is such a pull-back by Belyi functions (see $[\mathbf{1 6}, \mathbf{2 6}]$ ). By means of Grothendieck correspondence between Belyi pairs and dessin d'enfants, Litcanu [16] first showed how $P L_{n}(N)$ can be counted by using the combinatorics of dessins. Later by using this technique, Dahmen [15] obtained the explicit formula successfully:

$$
P L_{n}(N)=\left\{\begin{array}{l}
0 \quad \text { if } N \in\{1,2\} \\
\frac{n(n+1)}{12}(\Psi(N)-3 \phi(N))+\frac{2}{3} \varepsilon_{n}(N) \text { otherwise, }
\end{array}\right.
$$

where $\varepsilon_{n}(N)$ is defined in (5.24) below,

$$
\begin{aligned}
\phi(N) & :=\#\{k \in \mathbb{Z} \mid \operatorname{gcd}(k, N)=1,0 \leq k<N\} \\
& =N \prod_{p \mid N, p \text { prime }}\left(1-\frac{1}{p}\right)
\end{aligned}
$$

is the Euler function ( $\operatorname{set} \phi(N)=0$ if $N \notin \mathbb{N}$ ), and

$$
\Psi(N):=\# \mathcal{Q}(N)=N^{2} \prod_{p \mid N, p \text { prime }}\left(1-\frac{1}{p^{2}}\right) .
$$

Here $\mathcal{Q}(N)$ is the set of $N$-torsion points of exact order $N$ defined by

$$
\mathcal{Q}(N):=\left\{\left(\frac{k_{1}}{N}, \frac{k_{2}}{N}\right) \mid \operatorname{gcd}\left(k_{1}, k_{2}, N\right)=1,0 \leq k_{1}, k_{2}<N\right\} .
$$

However, differently from $P L_{n}(N), L_{n}(N)$ can not be counted by means of dessins and seeking the explicit formula of $L_{n}(N)$ remains a long-standing challenging problem. It is not difficult to see that, when monodromy group 
$M \simeq D_{N}$, then the projective monodromy group $P M \simeq D_{N / 2}$ if $N$ is even and $P M \simeq D_{N}$ if $N$ is odd. Thus (cf. [4])

$$
P L_{n}(N)=\left\{\begin{array}{l}
L_{n}(N)+L_{n}(2 N) \text { if } N \text { is odd, } \\
L_{n}(2 N) \text { if } N \text { is even. }
\end{array}\right\} .
$$

In 2003 Dahmen and Beukers proposed the following conjecture (see [4, Conjecture 73]):

Conjecture 5.5 (Dahmen-Beukers conjecture). For every $n \in \mathbb{N}$ and $N \in \mathbb{N}_{\geq 3}$,

$$
\begin{array}{r}
L_{n}(N)=\frac{1}{2}\left(\frac{n(n+1) \Psi(N)}{24}-\left[a_{n} \phi(N)+b_{n} \phi\left(\frac{N}{2}\right)\right]\right) \\
+\frac{2}{3} \varepsilon_{n}(N),
\end{array}
$$

where

$$
a_{2 n}=a_{2 n+1}=n(n+1) / 2, \quad b_{2 n}=b_{2 n-1}=n^{2},
$$

and

$$
\varepsilon_{n}(N)= \begin{cases}1 & \text { if } N=3 \text { and } n \equiv 1 \bmod 3 \\ 0 & \text { otherwise }\end{cases}
$$

This conjecture was proved by Dahmen himself $[\mathbf{1 5}, \mathbf{1 6}]$ for two cases: (i) $n \in \mathbb{N}$ and $4 \mid N$ (this follows directly from (5.17) and (5.21)); (ii) $n \in\{1,2,3\}$ and $N \in \mathbb{N}_{\geq 3}$. Recently, the case $n=4$ and $N \in \mathbb{N}_{\geq 3}$ was proved by Y.C. Chou [27, Appendix A]. As far as we know, the general case $n \geq 5$ and $4 \backslash N$ still remains open. In this paper, we confirm Dahmen-Beukers conjecture completely. See $\S 8$.

\subsection{Generalized Lamé equation and the monodromy group.} Once we knew how to explicitly express the monodromy group for (5.2), it is natural to study the problem of isomonodromy by deforming the moduli parameter $\tau$. For this purpose, we consider the following generalized Lamé equation:

$$
\frac{\mathrm{d}^{2} y}{\mathrm{~d} z^{2}}=I(z) y
$$

where

$$
\begin{aligned}
I(z)= & n(n+1) \wp(z)+\frac{3}{4}(\wp(z+\dot{p})+\wp(z-p)) \\
& +A(\zeta(z+p)-\zeta(z-p))+B, p \notin E_{\tau}[2],
\end{aligned}
$$

where $n \in \mathbb{N} \cup\{0\}$. Note that the local exponent at $p$ are $\frac{1}{2}$ and $-\frac{3}{2}$. Thus, the difference of the two local exponents is an integer. At this situation, the singular points $\pm p$ are called apparent if all solutions of (5.25) have no logarithmic singularity at $\pm p$. Throughout the paper, we always assume

$$
\pm p \text { are apparent singularities of (5.25). }
$$


Under the assumption of (5.27), the two constants $A$ and $B$ are not independent.

Lemma $5.6([\mathbf{7}])$. Suppose (5.27) holds. Then

$$
B=A^{2}-\zeta(2 p) A-\frac{3}{4} \wp(2 p)-n(n+1) \wp(p) .
$$

REMARK 5.7. Let $\rho$ be the monodromy representation of (5.25):

$$
\pi_{1}\left(E_{\tau} \backslash\{ \pm p\}, *\right) \rightarrow \mathrm{SL}(2, \mathbb{C}) .
$$

Let $\gamma_{ \pm}$be the simple loop in $\pi_{1}\left(E_{\tau} \backslash\{ \pm p\}, *\right)$ such that $\gamma_{ \pm}$encircles and $\pm p$ once. Then

$$
\rho\left(\gamma_{ \pm}\right)=-I_{2 \times 2}
$$

where $I_{2 \times 2}$ is the identity matrix of two by two. Therefore by ignoring $\rho\left(\gamma_{ \pm}\right)$, the monodromy representative is reduced to a homomorphism from $\pi_{1}\left(E_{\tau}\right)$ to $\mathrm{SL}(2, \mathbb{C})$, which implies the monodromy group of $(5.25)$ is always Abelian. Hence the monodromy representation is always reducible.

Next we want to discuss how to compute the monodromy transformation $\rho\left(\ell_{i}\right), i=1,2$, where $\ell_{i}$ are fundamental cycles in $\pi_{1}\left(E_{\tau}\right)$. Similar to (5.13), we consider the following ansatz for (5.25):

$$
y_{a}(z) \doteqdot e^{\sum_{i=1}^{n+1} \zeta\left(a_{i}\right) z} \frac{\prod_{i=1}^{n+1} \sigma\left(z-a_{i}\right)}{\sigma(z)^{n} \sqrt{\sigma(z+p) \sigma(z-p)}} .
$$

When $n=0, a=a_{1} \in E_{\tau}$ and the coefficient $A$ can be written in terms of $p$ and $a$.

TheOREM 5.8. [8] Suppose the potential I $(z)$ of (5.25) is given by (5.26) with $n=0$ and $p \notin E_{\tau}[2]$. Then $y_{a}$ of (5.28) is a solution of (5.25) iff

$$
A=\frac{1}{2}(\zeta(a+p)-\zeta(a-p)-\zeta(2 p)) .
$$

Moreover, the monodromy $\rho\left(\ell_{i}\right)$ is given by

$$
\rho\left(\ell_{1}\right)=\left(\begin{array}{cc}
e^{-2 \pi i s} & 0 \\
0 & e^{2 \pi i s}
\end{array}\right), \rho\left(\ell_{2}\right)=\left(\begin{array}{cc}
e^{2 \pi i r} & 0 \\
0 & e^{-2 \pi i r}
\end{array}\right),
$$

where $(r, s)$ is determined by

$$
\begin{aligned}
& r+s \tau=a, \\
& r \eta_{1}(\tau)+s \eta_{2}(\tau)=\zeta(a+p)+\zeta(a-p) .
\end{aligned}
$$

For $n \geq 1$, the ansatz plays also important roles for calculating the monodromy representation. 
TheOREM 5.9. Let $a=\left(a_{1}, \ldots, a_{n}\right) \in E_{\tau}^{n+1},\left[a_{i}\right] \notin\{ \pm p\}$, and $n \geq 1$. Then $y_{a}$ satisfies (5.25) iff

$$
\begin{gathered}
2 \sum_{j \neq i}^{n+1}\left(\zeta\left(a_{i}-a_{j}\right)+\zeta\left(a_{j}\right)-\zeta\left(a_{i}\right)\right) \\
=\zeta\left(a_{i}+p\right)+\zeta\left(a_{i}-p\right)-2 \zeta\left(a_{i}\right) \text { and } \\
\sum_{i=1}^{n+1} \zeta\left(a_{i}\right)=\frac{1}{2} \sum_{i=1}^{n+1}\left(\zeta\left(a_{i}+p\right)+\zeta\left(a_{i}-p\right)\right) .
\end{gathered}
$$

Moreover, A satisfies

$$
A=\frac{1}{2} \sum_{i=1}^{n+1}\left(\zeta\left(a_{i}+p\right)-\zeta\left(a_{i}-p\right)\right)-\frac{1}{2} \zeta(2 p) .
$$

Note that if $a$ satisfies (5.31), then $-a$ also satisfies (5.31). Clearly, $A$ also satisfies (5.32) with $-a$, that is, $y_{-a}$ is also a solution of (5.25) with the same coefficients $A$ and $B$. Moreover, it can be proved that either

$$
\begin{aligned}
& \left\{a_{1}, \ldots, a_{n+1}\right\} \cap\left\{-a_{1},-a_{2}, \ldots,-a_{n+1}\right\}=\emptyset \text { or } \\
& \left\{a_{1}, \ldots, a_{n+1}\right\}=\left\{-a_{1},-a_{2}, \ldots,-a_{n+1}\right\} .
\end{aligned}
$$

Let $(r, s) \in \mathbb{C}^{2}$ satisfy

$$
\left\{\begin{array}{l}
\sum_{i=1}^{n+1} a_{i}=r+s \tau \\
\sum_{i=1}^{n+1} \zeta\left(a_{i}\right)=r \eta_{1}(\tau)+s \eta_{2}(\tau) .
\end{array}\right.
$$

Then $(r, s)$ also are related to the monodromy data of (5.7). Let $\rho$ be the monodromy representation of $\pi_{1}\left(E_{\tau}\right)$ to $\mathrm{SL}(2, \mathbb{C})$, and $\ell_{i}, i=1,2$, are the fundamental cycles of $E_{\tau}$.

Theorem 5.10. [8] Let $a=\left(a_{1}, \ldots, a_{n+1}\right) \in E_{\tau}^{n+1}, A$ and $B \in \mathbb{C}$ such that (5.31), (5.32) and (4.33b) holds. Then GLE (5.25) is completely reducible, i.e., $y_{a}$ and $y_{-a}$ are linearly independent and the monodromy representation is given by

$$
\begin{aligned}
& \rho\left(\ell_{1}\right)^{*}\left(\begin{array}{c}
y_{a} \\
y_{-a}
\end{array}\right)=\left(\begin{array}{cc}
e^{2 \pi i s} & 0 \\
0 & e^{-2 \pi i s}
\end{array}\right)\left(\begin{array}{c}
y_{a} \\
y_{-a}
\end{array}\right), \\
& \rho\left(\ell_{2}\right)^{*}\left(\begin{array}{c}
y_{a} \\
y_{-a}
\end{array}\right)=\left(\begin{array}{cc}
e^{2 \pi i r} & 0 \\
0 & e^{-2 \pi i r}
\end{array}\right)\left(\begin{array}{c}
y_{a} \\
y_{-a}
\end{array}\right),
\end{aligned}
$$

where $(r, s) \in \mathbb{C}^{2}$ satisfies (5.34). Furthermore, $(r, s) \notin \frac{1}{2} \mathbb{Z}^{2}$.

REMARK 5.11. The GLE (5.25) such that the hypothesis of Theorem 5.10 holds dose not cover all the possible $(A, B)$. In [8], we proved that the set of exceptional pair $(A, B)$ is a finite set. Indeed, the classification of GLE (5.25) is more complicated than the Lamé equation (5.2). 
Similar to the Lamé equations, the GLE (5.25) also has a connection with the mean field equation,

$$
\Delta u+e^{u}=8 \pi n \delta_{0}+4 \pi\left(\delta_{p}+\delta_{-p}\right) \text { in } E_{\tau} .
$$

By the Liouville theorem, for any solution $u$ of (5.35), there exists an meromorphic function $f(z)$ in $\mathbb{C}$ such that (5.4) holds.

Assume $u(z)$ is an even solution. Then

$$
\begin{aligned}
u_{z z}-\frac{1}{2} u_{z}^{2}= & -2\left(n(n+1) \wp(z)+\frac{3}{4}(\wp(z+p)+\wp(z-p))\right) \\
& +A(\zeta(z+p)-\zeta(z-p)+B) \doteqdot I(z)
\end{aligned}
$$

and due to (5.4) the Schwarz derivative $\{f, z\}$ also satisfies

$$
\{f, z\}=u_{z z}-\frac{1}{2} u_{z}^{2}=-2 I(z) .
$$

By this identity, Proposition 5.1 implies that the developing map $f$ can be written as a ratio of two independent solutions of $(5.25)$, where $I(z)=$ $u_{z z}-\frac{1}{2} u_{z}^{2}$.

Proposition 5.12. [8] Let GLE (5.25) come from the mean field equation (5.35). Then $\pm p$ are apparent singular points for (5.25).

Proof. Suppose that (5.25) has solutions with logarithmic singularity at $[p]$. Then the local monodromy matrix of $(5.25)$ at $[p]$ is $\left(\begin{array}{cc}-1 & D \\ 0 & -1\end{array}\right)$ for some $D \neq 0$. Since near $p, f\left(p+(z-p) e^{2 \pi i}\right)$ (i.e., the analytic continuation of $f$ along a loop encircling $p$ counter-clockwise) is also a developing map of the same $u$, there exists $M \in \operatorname{PSU}(2)$ such that $f\left(p+(z-p) e^{2 \pi i}\right)=$ $M \cdot f(z)$ holds near $p$. Because Proposition 5.1 shows that $f$ is a ratio of two linearly independent solutions of (5.25), there is $\gamma \in \operatorname{SL}(2, \mathbb{C})$ such that $\gamma\left(\begin{array}{cc}-1 & D \\ 0 & -1\end{array}\right) \gamma^{-1}= \pm M$ (because $M=-M$ in $\left.\operatorname{PSU}(2)\right)$. Since $\pm M \in$ $\operatorname{PSU}(2)$ and $\gamma \in \mathrm{SL}(2, \mathbb{C})$, a direct computation gives $D=0$, which yields a contradiction.

After a normalization, $f$ satisfies also (5.6)

$$
f(z+1)=e^{2 \pi i \theta_{1}} f(z) \text { and } f(z+\tau)=e^{2 \pi i \theta_{2}} f(z), \theta_{i} \in \mathbb{R} .
$$

The identity (5.6) shows that the projective monodromy representation of $f$ is unitary. In fact under this normalization, we can show

$$
f=\frac{y_{a}(z)}{y_{-a}(z)},
$$

where $y_{a}$ is given by (5.28). Again the unitary monodromy yields that the "rescaled" $u_{\lambda}$,

$$
u_{\lambda}(z)=\log \frac{8 e^{2 \lambda}\left|f^{\prime}(z)\right|^{2}}{\left(1+e^{2 \lambda}|f(z)|^{2}\right)^{2}},
$$


is also a solution of (5.35). So the blowup set of $u_{\lambda}$ consists of the zeros of $f$ (the poles) as $\lambda \rightarrow+\infty(\lambda \rightarrow-\infty)$.

How many blowup points might $u_{\lambda}$ have? From the PDE result (see $[19]), u_{\lambda}$ never blows up at 0 . But they might blow up at $p$ or $-p$. Here, we assume

$$
\begin{aligned}
& \text { The one parameter family of solutions } u_{\lambda} \\
& \text { does not blow up at } \pm p \text {. }
\end{aligned}
$$

Under the assumption (5.37), the blowup set consists of $n+1$ points. Hence $f$ has $n+1$ distinct simple zeros $a_{1}, \ldots, a_{n+1}$ in $E_{\tau}$, similarly $n+1$ distinct poles $b_{1}, \ldots, b_{n+1}$. Moreover, we could prove $0, \pm p \notin\left\{a_{1}, \ldots, a_{n+1}\right\} \cup$ $\left\{b_{1}, \ldots, b_{n+1}\right\}$, if (5.36) holds. Moreover, we have

$$
\left\{b_{1}, \ldots, b_{n+1}\right\}=\left\{-a_{1},-a_{2}, \ldots,-a_{n+1}\right\} .
$$

Since $a_{1}, \ldots, a_{n+1}$ are blowup points of $u_{\lambda}$, their location can be determined by a equation similar to (3.1) by the Pohozaev identity, which turns out to be (5.31). After that the coefficients $A$ and $B$ can be determined by (5.32) and Lemma 5.6 (by Proposition 5.12). Applying Theorem 5.10 we have

THEOREM 5.13. [8] (5.35) has even solution such that (5.37) holds iff there exists $\left(a_{1}, \ldots, a_{n+1}\right) \in E_{\tau}^{n+1},\left[a_{i}\right] \notin\{ \pm p\}$ such that the hypothesis of Theorem 5.10 holds with $(r, s) \in \mathbb{R}^{2} \backslash \frac{1}{2} \mathbb{Z}^{2}$, where $(r, s)$ is determined by (5.34).

Now we consider the PDE (5.35) with the case $p=p(\tau)$, i,e, the vortex point $p$ is a holomorphic function $\tau$, such that the projective monodromy group of its developing map is a constant independent of $\tau$. This is an isomonodromic definition, and naturally it is related to the Painlevé equations, the main subject of the next section.

\section{Painlevé VI equation}

6.1. Elliptic forms. In literature, a nonlinear differential equation in one complex variable is said to possess the Painlevé property if its solutions have neither movable branch points nor movable essential singularities. For the class of second order differential equations

$$
\lambda^{\prime \prime}(t)=F\left(t, \lambda, \lambda^{\prime}\right), \quad t \in \mathbb{C P}^{1}
$$

where $F\left(t, \lambda, \lambda^{\prime}\right)$ is meromorphic in $t$ and rational in both $\lambda$ and $\lambda^{\prime}$, Painlevé (later completed by Gambier) obtained the classification of those nonlinear ODEs which possess the Painlevé property. They showed that there were fifty canonical equations of the form (6.1) with this property, up to Möbius transformations. Furthermore, of these fifty equations, forty-four are either integrable in terms of previously known functions (such as elliptic functions), equivalent to linear equations, or are reduced to one of six new nonlinear ODEs which define new transcendental functions (see e.g. [17]). These six nonlinear ODEs are called Painlevé equations. Among them, Painlevé VI is often considered to be the master equation, because others can be obtained 
from Painlevé VI by the confluence. Due to its connection with many different disciplines in mathematics and physics, Painlevé VI has been extensively studied in the past several decades. See $[\mathbf{5}, \mathbf{1 8}, \mathbf{1 9}, \mathbf{2 0}, \mathbf{2 3}, \mathbf{2 4}, \mathbf{2 5}, \mathbf{2 9}, \mathbf{3 1}$, 32, 33] and the references therein.

Painlevé VI with four free parameters $(\alpha, \beta, \gamma, \delta)$ denoted by $\operatorname{PVI}(\alpha, \beta$, $\gamma, \delta)$ can be written as

$$
\begin{aligned}
\frac{\mathrm{d}^{2} \lambda}{\mathrm{d} t^{2}}= & \frac{1}{2}\left(\frac{1}{\lambda}+\frac{1}{\lambda-1}+\frac{1}{\lambda-t}\right)\left(\frac{\mathrm{d} \lambda}{\mathrm{d} t}\right)^{2} \\
& -\left(\frac{1}{t}+\frac{1}{t-1}+\frac{1}{\lambda-t}\right)\left(\frac{\mathrm{d} \lambda}{\mathrm{d} t}\right) \\
& +\frac{\lambda(\lambda-1)(\lambda-t)}{t^{2}(t-1)^{2}}\left[\alpha+\beta \frac{t}{\lambda^{2}}+\gamma \frac{t-1}{(\lambda-1)^{2}}+\delta \frac{t(t-1)}{(\lambda-t)^{2}}\right] .
\end{aligned}
$$

An important fact to the PVI (6.1) says that PVI (6.1) possesses the Painlevé property. Notice that the branch points of PVI (6.1) which do not depend on any particular solutions, consist of $\{0,1, \infty\}$ only. From the Painlevé property, any solution $\lambda(t)$ is a multi-valued meromorphic function in $\mathbb{C P}^{1} \backslash\{0,1, \infty\}$. To avoid the multi-valueness of $\lambda(t)$, it is better to lift solutions of (6.1) to its universal covering. It is known that the universal covering of $\mathbb{C P}^{1} \backslash\{0,1, \infty\}$ is $\mathbb{H}$. Then $t$ and solution $\lambda(t)$ can be lifted to $\tau$ and $p(\tau)$ respectively through the covering map by

$$
t(\tau)=\frac{e_{3}(\tau)-e_{1}(\tau)}{e_{2}(\tau)-e_{1}(\tau)} \text { and } \lambda(t)=\frac{\wp(p(\tau) \mid \tau)-e_{1}(\tau)}{e_{2}(\tau)-e_{1}(\tau)} .
$$

Then $p(\tau)$ satisfies the following elliptic form

$$
\frac{\mathrm{d}^{2} p(\tau)}{\mathrm{d} \tau^{2}}=\frac{-1}{4 \pi^{2}} \sum_{i=0}^{3} \alpha_{i} \wp^{\prime}\left(p(\tau)+\frac{\omega_{i}}{2} \mid \tau\right)
$$

where

$$
\left(\alpha_{0}, \alpha_{1}, \alpha_{2}, \alpha_{3}\right)=\left(\alpha,-\beta, \gamma, \frac{1}{2} \delta\right) \text {. }
$$

This elliptic form was already known to Painlevé [32]. For a modern proof, see $[\mathbf{2}, \mathbf{3 0}]$. The advantage of $(6.3)$ is that $\wp(p(\tau) \mid \tau)$ is single-valued for $\tau \in \mathbb{H}$, although $p(\tau)$ has a branch point at those $\tau_{0}$ such that $p\left(\tau_{0}\right) \in E_{\tau_{0}}[2]$.

6.2. Isomonodromy and Hamiltonian systems. Another important feature of (6.1) is that any solution $\lambda(t)$ (or $p(\tau))$ governs the isomonodromic deformation of some linear ODE (see $[\mathbf{1 9}, \mathbf{2 4}])$. There are several different types of linear ODE associated with the Painlevé VI. Here we only discuss the second order ODE associated with (6.1).

Consider a Heun second order equation with five regular points $\{0,1, \infty$, $t, \lambda\}$,

$$
y^{\prime \prime}(x)+p_{1}(x) y^{\prime}(x)+p_{1}(x) y(x)=0,
$$

with the following Riemann scheme 


$$
\left(\begin{array}{ccccc}
0 & 1 & t & \infty & \lambda \\
0 & 0 & 0 & \alpha & 0 \\
\theta_{1} & \theta_{2} & \theta_{3} & \alpha+\theta_{\infty} & 2
\end{array}\right) .
$$

Here we always assume

$$
\theta_{i} \notin \mathbb{Z}, i=1,2,3, \infty \text { and } \lambda \text { is an apparent singular point. }
$$

The classical Fuchsian relation implies that

$$
\alpha=-\frac{1}{2}\left\{\sum_{i=1}^{3} \theta_{i}+\theta_{\infty}-1\right\} .
$$

The coefficients $p_{1}(x)$ and $p_{2}(x)$ are uniquely determined by the conditions (6.5) and (6.6) up to two constants $\mu$ and $K$ :

$$
\mu=\operatorname{Res}_{x=\lambda} p_{2}(x) \text { and } K=-\operatorname{Res}_{x=\lambda} p_{2}(x) .
$$

Indeed, a straightforward computation shows

$$
\begin{aligned}
& p_{1}(x)=\sum_{i=1}^{3} \frac{1-\theta_{i}}{x-t_{i}}-\frac{1}{x-\lambda} \text { and } \\
& p_{2}(x)=\frac{\kappa}{x(x-1)}-\frac{t(t-1) K}{x(x-1)(x-t)}+\frac{\lambda(\lambda-1) \mu}{x(x-1)(x-\lambda)},
\end{aligned}
$$

where

$$
t_{1}=0, t_{2}=1, t_{3}=t
$$

and

$$
\kappa=\frac{1}{4}\left[\left(\sum_{i=1}^{3} \theta_{i}-1\right)^{2}-\theta_{\infty}^{2}\right] .
$$

Now suppose that Heun ODE (6.4) with the fixed Riemann scheme (6.5) keep the same monodromy representation while $t$ is deformed.

TheOREM 6.1. Suppose (6.6). Then the monodromy representation of (6.4) with fixed Riemann scheme (6.5) is invariant under the deformation of $t$ iff $(\lambda(t), \mu(t))$ satisfies the Hamiltonian system

$$
\begin{cases}\frac{\mathrm{d} \lambda(t)}{\mathrm{d} t} & =\frac{\partial}{\partial \mu} K(\lambda, \mu, t), \\ \frac{\mathrm{d} \mu(t)}{\mathrm{d} t} & =-\frac{\partial}{\partial \lambda} K(\lambda, \mu, t),\end{cases}
$$

where

$$
\begin{aligned}
K(\lambda, \mu, t)= & \frac{1}{t(t-1)}\left[\lambda(\lambda-1)(\lambda-t) \mu^{2}-\left\{\theta_{2}(\lambda-1)(\lambda-t)\right.\right. \\
& \left.\left.+\theta_{3} \lambda(\lambda-t)+\left(\theta_{1}-1\right) \lambda(\lambda-1)\right\} \mu+\kappa \lambda\right] .
\end{aligned}
$$

The Hamiltonian system (6.11) with $K$ given by (6.12) plays an important role in the application of the Painlevé VI. Indeed, this Hamiltonian system is equivalent to the Painlevé equation, as shown in the following theorem. 
TheOREM 6.2. [24] Suppose $\lambda(t)$ and $\mu(t)$ satisfies the Hamiltonian system (6.11) with the Hamiltonian $K(\lambda, \mu, t)$ iff $\lambda(t)$ is a solution of Painlevé $V I(6.1)$.

The parameters of the Hamiltonian system and the Painlevé VI has the relation:

$$
(\alpha, \beta, \gamma, \delta)=\left(\frac{1}{2} \theta_{4}^{2},-\frac{1}{2} \theta_{1}^{2}, \frac{1}{2} \theta_{2}^{2}, \frac{1}{2}\left(1-\theta_{3}^{2}\right)\right) .
$$

We remark that $\mu$ can be solved in terms of $\lambda$ and $\lambda^{\prime}$ from the first equation of (6.11) (Note that $\frac{\partial \mathcal{K}}{\partial \mu}$ is linear in $\mu$ ). Thus, the necessary part of Theorem 6.2 says that $\mu(t)$ satisfies the second equation of $(6.11)$ if $\lambda(t)$ satisfies (6.1). The proof of Theorem 6.2 is a direct computation only. We note that the Painlevé VI (6.1) and the Hamiltonian system are equivalent without any condition. Nevertheless, the isomonodromy of (6.4) is equivalent to the Hamiltonian under the non-resonant condition $\theta_{i} \notin \mathbb{Z}$. We refer the readers to $[\mathbf{2 4}]$ for the excellent introduction of Painlevé equations and the proof of Theorem 6.1. and Theorem 6.2.

For the elliptic form (6.3) $\mathrm{PVI}\left(\alpha_{i}\right)$, any solution $p(\tau)$ of (6.3) governs the isomonodromic deformation of a GLE:

$$
y^{\prime \prime}=I(z \mid \tau) y,
$$

where

$$
\begin{aligned}
I(z \mid \tau)= & \sum n_{i}\left(n_{i}+1\right) \wp\left(z-\frac{\omega_{i}}{2}\right)+\frac{3}{4}(\wp(z+p)-\wp(z-p)) \\
& +A(z)(\zeta(z+p)-\zeta(z-p))+B,
\end{aligned}
$$

and the non-resonant condition is

$$
n_{i} \notin \frac{1}{2}+\mathbb{Z} .
$$

The Hamiltonian system is

$$
\begin{cases}\frac{\mathrm{d} p(\tau)}{\mathrm{d} \tau} & =\frac{\partial \mathcal{K}}{\partial A}, \\ \frac{\mathrm{d} A(\tau)}{\mathrm{d} \tau} & =-\frac{\partial \mathcal{K}}{\partial p},\end{cases}
$$

where the Hamiltonian $\mathcal{K}$ is given by

$$
\begin{gathered}
\mathcal{K}(p, A, \tau)=\frac{-i}{4 \pi}\left[A^{2}+\left(2 p \eta_{1}(\tau)-\zeta(2 p \mid \tau)\right) A-\frac{3}{4} \wp(2 p \mid \tau)\right. \\
\left.-\sum_{k=0}^{3} n_{k}\left(n_{k}+1\right) \wp\left(p+\frac{\omega_{k}}{2} \mid \tau\right)\right] .
\end{gathered}
$$

The equivalence of isomonodromy, the Hamiltonian and the elliptic form (6.4) are given in the following theorem:

THEOREM 6.3.

(i) Suppose $n_{i} \notin \frac{1}{2}+\mathbb{Z}, i=0,1,2,3$. Then the monodromy representation of (6.13) is invariant under the deformation of $\tau$ iff $(p(\tau), A(\tau))$ satisfies the Hamiltonian system (6.15). 
(ii) The pair $(p(\tau), A(\tau))$ satisfies the Hamiltonian system (6.15) iff $p(\tau)$ satisfies the elliptic form (6.3). Moreover the relation of parameters of (6.3) and (6.13) is

$$
\alpha_{i}=\frac{1}{2}\left(\frac{1}{2}+n_{i}\right)^{2}, \quad 0 \leq i \leq 3 .
$$

6.3. Hitchin's theorem and applications. The expression of the elliptic form (6.3) looks beautiful and more attractive than (6.1). For example, if $\alpha_{i}=0, \forall i$, then $p(\tau)$ is linear in $\tau$, i.e., $p(\tau)=r+s \tau$. The corresponding $\lambda(t)$ (see $(6.1)$ ) is called the Picard solution. This is the first example of solutions with explicit expression. Another remarkable example is the Hitchin theorem [22] for PVI with the parameters: $\alpha_{i}=\frac{1}{8}$.

Theorem 6.4 (Hitchin). [22] For any $(r, s) \in \mathbb{C}^{2} \backslash \frac{1}{2} \mathbb{Z}^{2}$, let $p_{r, s}(\tau)$ be given by

$$
\begin{aligned}
\wp\left(p_{r, s}(\tau) \mid \tau\right)= & \wp(r+s \tau \mid \tau) \\
& +\frac{\wp^{\prime}(r+s \tau \mid \tau)}{2\left(\zeta(r+s \tau \mid \tau)-r \eta_{1}(\tau)-s \eta_{2}(\tau)\right)} .
\end{aligned}
$$

Then $p_{r, s}(\tau)$ is a solution to the elliptic form (6.3) with $\alpha_{i}=\frac{1}{8}, i=0,1,2,3$.

There are several ways to prove the Hitchin theorem. One is to use Theorem 6.1 to find that the monodromy representation of (6.13) with $n=0$ is generated by $\left(\begin{array}{cc}e^{2 \pi i s} & 0 \\ 0 & e^{-2 \pi i s}\end{array}\right)$ and $\left(\begin{array}{cc}e^{2 \pi i r} & 0 \\ 0 & e^{-2 \pi i r}\end{array}\right)$. This calculation of the monodromy representation has been done in $\S 5$. In (5.28), $y_{ \pm a}(z)$ are two independent solutions of (5.10) iff $a \notin E_{\tau}[2]$,

$$
\begin{aligned}
A & =\frac{1}{2}[\zeta(p+a)-\zeta(p-a)-\zeta(2 p)], \\
B & =A^{2}-\zeta(2 p) A-\frac{3}{4} \wp(2 p) .
\end{aligned}
$$

The monodromy data of (5.25) with (6.19) and $n=0$ are provided by the pair $(\mathrm{r}, \mathrm{s})$ solving the $2 \times 2$ linear system

$$
\begin{aligned}
& r+s \tau=a, \\
& 2\left(r \eta_{1}(\tau)+s \eta_{2}(\tau)\right)=\zeta(a+p)+\zeta(a-p) .
\end{aligned}
$$

See Theorem 5.8 .

Recall the addition theorem

$$
\frac{\wp^{\prime}(a)}{\wp(a)-\wp(p)}=\zeta(a+p)+\zeta(a-p)-2 \zeta(a) .
$$

Proof of Hitchin theorem. Suppose $p_{r, s}(\tau)$ is a smooth branch given by (6.18). Without loss of generality, we may assume $p_{r, s}\left(\tau_{0}\right) \notin E_{\tau_{0}}[2]$. Then, $p_{r, s}(\tau)$ is uniquely determined by (6.18) and $p_{r, s}(\tau) \rightarrow p_{r, s}\left(\tau_{0}\right)$ as $\tau \rightarrow \tau_{0}$. Let $a(\tau)=r+s \tau$ and $(A(\tau), B(\tau))$ are given by (6.19). Let (6.13) be the GLE with $\left(p_{r, s}(\tau), A(\tau), B(\tau)\right)$ and the monodromy data are given by $(\bar{r}, \bar{s})$ given by $(6.20)$ with $a=a(\tau)$ and $p=p_{r, s}(\tau)$. In a small neighborhood 
of $\tau_{0} \notin E_{\tau}[2],(6.18)$ and the addition theorem implies $\zeta(a(\tau)+p(\tau))+\zeta(a(\tau)-p(\tau))-2 \zeta(a(\tau))=2\left(r \eta_{1}(\tau)+s \eta_{2}(\tau)-2 \zeta(a(\tau))\right)$

which implied

$$
\zeta(a+p)+\zeta(a-p)=r \eta_{1}(\tau)+s \eta_{2}(\tau) .
$$

Together with $a(\tau)=r+s \tau$, it yields $(\bar{r}, \bar{s})=(r, s)$. Therefore, the monodromy representation of GLE (6.13) with $(A(\tau), B(\tau))$ given by $(6.19)$ is invariant under the deformation of $\tau$. By Theorem $6.3, p_{r, s}(\tau)$ satisfies the Painlevé VI equation with $\alpha_{i}=\frac{1}{8}, \forall i$.

Conversely if $p(\tau)$ is a solution of $\operatorname{PVI}\left(\frac{1}{8}\right)$ and assume the monodromy representation associated with GLE (6.13) is completely reducible, then our previous work $[\mathbf{1 0}]$ shows

$$
\wp(p(\tau))=\wp\left(p_{r, s}(\tau)\right) \text { for some } r, s \in \mathbb{C} \backslash \frac{1}{2} \mathbb{Z}^{2} .
$$

Thus, the formula (6.18) gives all the completely reducible solutions of $\operatorname{PVI}\left(\frac{1}{8}\right)$.

It is remarkable that the denominator of (6.18) is the Hecke form $Z_{r, s}(\tau)$, which is discussed in previous sections. The Painlevé property says that if $\tau_{0}$ is a pole of $\wp(p(\tau) \mid \tau)$, then $\tau_{0}$ is a simple pole. This Painlevé property implies

Theorem 6.5. [10] Suppose $(r, s) \in \mathbb{C}^{2} \backslash \frac{1}{2} \mathbb{Z}^{2}$. Then $Z_{r, s}(\tau)$ has only simple zeros.

PROOF. First, we note that the situations $r+s \tau \in E_{\tau}[2]$ and $Z_{r, s}(\tau)=0$ can not occur simultaneously. If not, then there are $\tau_{0}$ and $m, n \in \mathbb{Z}$ such that $r+s \tau_{0}=m+n \tau_{0}+\frac{\omega}{2}$, where $\omega \in \Lambda_{\tau}$, and also $\zeta\left(r+s \tau_{0}\right)=r \eta_{1}\left(\tau_{0}\right)+s \eta_{2}\left(\tau_{0}\right)$. Without loss of generality, we might assume $\omega=\omega_{1}$. The other cases can be proved similarly.

The zero of denominator of (6.18) implies

$$
\begin{aligned}
\frac{1}{2} \eta_{1}\left(\tau_{0}\right) & =\zeta\left(\frac{\omega_{1}}{2}\right)=\zeta\left((r-m)+(s-n) \tau_{0}\right) \\
& =\zeta\left(r+s \tau_{0}\right)-m \eta_{1}\left(\tau_{0}\right)-n \eta_{2}\left(\tau_{0}\right) \\
& =(r-m) \eta_{1}\left(\tau_{0}\right)+(s-n) \eta_{2}\left(\tau_{0}\right) .
\end{aligned}
$$

Therefore, we have

$$
\begin{gathered}
\left(r-m-\frac{1}{2}\right)+(s-n) \tau_{0}=0, \\
\left(r-m-\frac{1}{2}\right) \eta_{1}\left(\tau_{0}\right)+(s-n) \eta_{2}\left(\tau_{0}\right)=0,
\end{gathered}
$$

which implies $\left(r-m-\frac{1}{2}\right)=0$ and $s=n$ because of the matrix $\left(\begin{array}{cc}1 & \tau \\ \eta_{1}(\tau) & \eta_{2}(\tau)\end{array}\right)$ is non-degenerate for any $\tau$ due to the Legendre relation. Obviously it contradicts to the assumption $(r, s) \notin \frac{1}{2} \mathbb{Z}^{2}$. 
Now suppose $Z_{r, s}\left(\tau_{0}\right)=0$, which implies $\wp\left(p\left(\tau_{0}\right)\right)=\infty$ by $(6.18)$ because $\wp\left(r+s \tau_{0}\right) \neq 0$. Consider the transformation $\tau_{0} \mapsto t_{0}$ via $(6.2)$. Then by the Painlevé property, we know that $\lambda(t)$ has a pole at $t=t_{0} \notin\{0,1, \infty\}$. By substituting the local expansion of $\lambda(t)$ at $t=t_{0}$ into (6.1), it is easy to prove that the order of pole at $t=t_{0}$ is 1 , which implies the zero of $Z_{r, s}$ at $\tau=\tau_{0}$ is simple.

Theorem 6.5 is the first application of the Painlevé property. In the next subsection, we will give another application of this property.

6.4. The smoothness of the degenerate curves. Next we are going to discuss another application of $\operatorname{PVI}\left(\frac{1}{8}\right)$. As we explained, (6.18) consists of only completely reducible solutions of $\mathrm{PVI}\left(\frac{1}{8}\right)$. The other non-completely reducible solutions come from the solutions of Riccati equations.

THEOREM 6.6. [10] There are four Riccati equations reduced from Painlevé VI with $(\alpha, \beta, \delta, \gamma)=\left(\frac{1}{8},-\frac{1}{8}, \frac{1}{8}, \frac{3}{8}\right)$. The complete list of solutions to these four Riccati equations are given by:

$$
\lambda_{C}(t)=\frac{\wp\left(p_{C}(\tau) \mid \tau\right)-e_{1}(\tau)}{e_{2}(\tau)-e_{1}(\tau)}, t=\frac{e_{3}(\tau)-e_{1}(\tau)}{e_{2}(\tau)-e_{1}(\tau)},
$$

where $p_{C}(\tau)$ is given by the following (i) and (ii).

(i) $\lambda_{C}(t)$ solves the Riccati equation

$$
\lambda^{\prime}(t)=-\frac{1}{2 t(t-1)}\left(\lambda^{2}-2 t \lambda+t\right)
$$

if and only if there exists constant $C \in \mathbb{C} \cup\{\infty\}$ such that

$$
\begin{aligned}
\wp\left(p_{C}(\tau) \mid \tau\right) & =\frac{\eta_{2}(\tau)-C \eta_{1}(\tau)}{C-\tau}=-\eta_{1}+\frac{2 \pi i}{\tau-C}, \\
\eta_{2} & =\tau \eta_{1}-2 \pi i .
\end{aligned}
$$

(ii) For $C \in \mathbb{C} \cup\{\infty\}$, we let

$$
\wp\left(p_{C}(\tau) \mid \tau\right)=\frac{e_{k}\left(C \eta_{1}(\tau)-\eta_{2}(\tau)\right)+\left(\frac{g_{2}}{4}-2 e_{k}^{2}\right)(C-\tau)}{C \eta_{1}(\tau)-\eta_{2}(\tau)+e_{k}(C-\tau)}, k=1,2,3
$$

Then $\lambda_{C}(t)$ satisfies the Riccati equation

$$
\begin{aligned}
& \lambda^{\prime}(t)=-\frac{1}{2 t(t-1)}\left(\lambda^{2}-2 t \lambda+t\right), \quad \text { for } k=1 \\
& \lambda^{\prime}(t)=-\frac{1}{2 t(t-1)}\left(\lambda^{2}-t\right), \quad \text { for } k=2 \\
& \lambda^{\prime}(t)=-\frac{1}{2 t(t-1)}\left(\lambda^{2}+2(t-1) \lambda+t\right), \quad \text { for } k=3 .
\end{aligned}
$$

Furthermore, such $\lambda_{C}(t)$ give all the solutions of these three Riccati equations in (ii).

A simple observation is that $C=\infty$ in (6.22) gives that 


$$
\lambda_{\infty}(t)=-\frac{\eta_{1}(\tau)+e_{1}(\tau)}{e_{2}(\tau)-e_{1}(\tau)}
$$

is a solution of $\mathrm{PVI}_{\left(\frac{1}{8},-\frac{1}{8}, \frac{1}{8}, \frac{3}{8}\right)}$. Since $\lambda_{\infty}(t), \lambda_{\infty}(t)-1$ and $\lambda_{\infty}(t)-t$ can have only simple zeros, a direct consequence is

TheOREM 6.7. [10] For fixed $k \in\{1,2,3\}$, the followings hold:

(i) Any zero of $\eta_{1}(\tau)+e_{k}(\tau)$ must be simple.

$$
\frac{\mathrm{d}}{\mathrm{d} \tau}\left(\left(\eta_{1}(\tau)-e_{k}(\tau)\right)^{-1}\right) \neq \frac{1}{2 \pi i} \quad \text { for any } \tau \in \mathbb{H} .
$$

(iii) $\tau-\frac{2 \pi i}{\eta_{1}(\tau)-e_{k}(\tau)}=\frac{\eta_{2}(\tau)-\tau e_{k}(\tau)}{\eta_{1}(\tau)-e_{k}(\tau)}$ is a locally one-to-one map from $\mathbb{H}$ to $\mathbb{C} \cup\{\infty\}$.

Proof. Recall

$$
t=t(\tau)=\frac{e_{3}(\tau)-e_{1}(\tau)}{e_{2}(\tau)-e_{1}(\tau)}
$$

Since $t^{\prime}(\tau) \neq 0$ for all $\tau \in \mathbb{H}$, the assertion (i) follows readily from the fact that $\lambda_{\infty}(t)($ for $k=1), \lambda_{\infty}(t)-1($ for $k=2)$ and $\lambda_{\infty}(t)-t$ (for $k=3$ ) can have only simple zeros.

For the assertion (ii), we note from the Legendre relation that $\lambda_{C}(t)$ defined by $(6.22)$ is

$$
\lambda_{C}(t)=\frac{\wp\left(p_{C}(\tau) \mid \tau\right)-e_{1}(\tau)}{e_{2}-e_{1}(\tau)}=\frac{-\eta_{1}(\tau)-e_{1}(\tau)+\frac{2 \pi i}{\tau-C}}{e_{2}(\tau)-e_{1}(\tau)} .
$$

Fix any $\tau_{0} \in \mathbb{H}$. If $\tau_{0}$ is a zero of $\eta_{1}+e_{1}$, then by (i) or Painlevé property of $\lambda_{\infty}(t), \tau_{0}$ is a simple pole. So $\tau-\frac{2 \pi i}{\eta_{1}(\tau)-e_{1}(\tau)}$ is locally one-one near $\tau_{0}$. So it suffices to consider the case $\eta_{1}\left(\tau_{0}\right)+e_{1}\left(\tau_{0}\right) \neq 0$. Then by letting

$$
C=\tau_{0}-\frac{2 \pi i}{\eta_{1}\left(\tau_{0}\right)-e_{k}\left(\tau_{0}\right)}
$$

we see that $t_{0}=t\left(\tau_{0}\right)$ is a zero of $\lambda_{C}(t)$. Since $\lambda_{C}(t)$ has only simple zeros, we have

$$
\left.\frac{\mathrm{d}}{\mathrm{d} \tau}\left(\eta_{1}(\tau)-e_{1}(\tau)-\frac{2 \pi i}{\tau-C}\right)\right|_{\tau=\tau_{0}} \neq 0 .
$$

This, together with $\eta_{1}\left(\tau_{0}\right)-e_{1}\left(\tau_{0}\right)-\frac{2 \pi i}{\tau_{0}-C}=0$, easily implies

$$
\frac{\mathrm{d}}{\mathrm{d} \tau}\left(\left(\eta_{1}(\tau)-e_{k}(\tau)\right)^{-1}\right) \neq \frac{1}{2 \pi i} .
$$

This proves (6.24) for $k=1$. Similarly, by considering $\lambda_{C}(t)-1$ and $\lambda_{C}(t)-t$, we can prove (6.24) for $k=2,3$. This prove the assertion (ii).

Finally, using the Legendre relation leads to

$$
\frac{\eta_{2}(\tau)-\tau e_{k}(\tau)}{\eta_{1}(\tau)-e_{k}(\tau)}=\tau-\frac{2 \pi i}{\eta_{1}(\tau)-e_{k}(\tau)} .
$$

Therefore, $\frac{\eta_{2}(\tau)-\tau e_{k}(\tau)}{\eta_{1}(\tau)-e_{k}(\tau)}$ is locally one-to-one. This completes the proof. 
We have already proved that the critical points of Green function $G(z \mid \tau)$ could bifurcate only at half periods. It is easy to see that the bifurcation could occur at $\frac{\omega_{i}}{2}$ iff the Hessian of $G(z \mid \tau)$ at $z=\frac{\omega_{i}}{2}$ vanishes. By a direct computation, we have

$$
\begin{aligned}
\operatorname{det} \mathrm{D}_{z}^{2} G\left(\frac{\omega_{k}}{2} \mid \tau\right)=- & \frac{1}{4 \pi^{2} \operatorname{Im} \tau}\left|\eta_{1}(\tau)+e_{k}(\tau)\right|^{2} \\
& \operatorname{Im}\left\{\tau-\frac{2 \pi i}{\eta_{1}(\tau)+e_{k}(\tau)}\right\} .
\end{aligned}
$$

Recall $C_{k}$ to be the curve of those $\tau \in F_{2}$ such that $\frac{\omega_{k}(\tau)}{2}$ is degenerate. Then Theorem 6.7 implies $C_{k}$ is smooth.

Corollary 6.8. [10] The degenerate curve $C_{k}, k=1,2,3$, is smooth.

To conclude this section, we state another application of Theorem 6.7.

Theorem 6.9. [10] For any fundamental domain $G$ of $\Gamma(2), \eta_{1}(\tau)+$ $e_{k}(\tau)$ has at most a zero in $F$.

Proof. Note that if $e_{k}(\tau)+\eta_{1}(\tau) \neq 0$, then $\tau \in C_{k}$ if and only if $\operatorname{Im}\left(\tau-\frac{2 \pi i}{e_{k}(\tau)+\eta_{1}(\tau)}\right)=0$. Theorem 6.7 says that $e_{k}(\tau)+\eta_{1}(\tau)$ has only simple zeros, and any zero of $e_{k}(\tau)+\eta_{1}(\tau)$ lies on the curve $C_{k}$.

Clearly $e_{k}(\tau)+\eta_{1}(\tau)$ is not a modular form. However, if $\tau^{\prime}=\gamma \cdot \tau$ for some $\gamma \in S L(2, \mathbb{Z})$, then $E_{\tau^{\prime}}$ is conformally equivalent to $E_{\tau}$ and transforms $C_{k}$ to another degenerate curve $C_{j}$. Therefore, without loss of generality, we may assume $k=1$.

From the map $z \rightarrow \frac{z}{c \tau+d}$, if $\gamma \in \Gamma_{0}(2)=\{\gamma \in \mathrm{SL}(2, \mathbb{Z}) \mid c \equiv 0(\bmod 2)\}$, the image of $C_{1}\left(F_{2}\right)$ is mapped to $C_{1}\left(F_{2}^{\prime}\right)$ for another fundamental domain $F_{2}^{\prime}:=\gamma\left(F_{2}\right)$. For example, if $\gamma=T S^{-1} T^{2} S^{-1}=\left(\begin{array}{ll}1 & -1 \\ 2 & -1\end{array}\right)$, i.e.

$$
\tau^{\prime}=\gamma \cdot \tau=\frac{\tau-1}{2 \tau-1}
$$

then $F_{2}^{\prime}$ is the domain bounded by 3 half circles:

$$
F_{2}^{\prime}=\left\{\tau \in \mathbb{H}|| \tau-\frac{1}{2}\left|\leq \frac{1}{2},\right| \tau-\frac{1}{4}\left|\geq \frac{1}{4},\right| \tau-\frac{3}{4} \mid \geq \frac{1}{4}\right\} .
$$

Noting that the curve $\left\{\tau|| \tau-\frac{1}{2} \mid=\frac{1}{2}\right\}$ is invariant under $\gamma$, the curves $\overline{C_{1}\left(F_{2}\right)}, \overline{C_{1}\left(F_{2}^{\prime}\right)}$ bound a simply connected domain $\mathcal{D}$ in

$$
F \doteqdot F_{2} \cup F_{2}^{\prime}=\left\{\tau \in \mathbb{H}|0 \leq \operatorname{Re} \tau \leq 1,| \tau-\frac{1}{4}\left|\geq \frac{1}{4},\right| \tau-\frac{3}{4} \mid \geq \frac{1}{4}\right\}
$$

where $\overline{\mathcal{D}} \cap \mathbb{R}=\{0,1\}$. Since $\Gamma_{0}(2)=\Gamma(2) \cup \gamma \Gamma(2), F_{2} \cup F_{2}^{\prime}$ is also a fundamental domain of $\Gamma(2)$. Note that for any $\tau \in \mathcal{D}$, the half period $\frac{1}{2}$ is a minimum point of $G(z \mid \tau)$ in $E_{\tau}$, and (6.25) yields that $\frac{1}{2}$ is actually a non-degenerate critical point of $G(z \mid \tau)$. Therefore $\operatorname{det} D_{z}^{2}\left(\frac{1}{2} \mid \tau\right)>0$ and then $\operatorname{Im}\left\{\tau-\frac{2 \pi i}{\eta_{1}+e_{1}}\right\}>0 \forall \tau \in D$. For simplicity of notation, we denote $\tau-\frac{2 \pi i}{\eta_{1}(\tau)+e_{1}(\tau)}$ by $f(\tau)$. Then Theorem 6.7 implies $f(\tau)$ is a locally one-one map from $\mathcal{D}$ to the lower half plane $\mathbb{C}_{-}=\{\kappa \mid \operatorname{Im} \kappa<0\}$. 
Obviously, the map $f(\tau)$ maps $\overline{C_{1}\left(F_{2}\right) \cup C_{1}\left(F_{2}^{\prime}\right)}$ to the real axis. By $[\mathbf{2 8}$, Theorem 1.6],

$$
C_{1}\left(F_{2}\right) \cap\left\{\tau \mid \operatorname{Re} \tau=\frac{1}{2}\right\}=\left\{\frac{1}{2}+i b_{1}\right\},
$$

where $b_{1} \in\left(\frac{1}{2}, \frac{\sqrt{3}}{2}\right)$ is the unique zero of the increasing function in $b$

$$
e_{1}+\eta_{1}-\frac{2 \pi}{b}
$$

along the vertical line $\frac{1}{2}+i b$. Similarly, $C_{1}\left(F_{2}^{\prime}\right) \cap\left\{\tau \mid \operatorname{Re} \tau=\frac{1}{2}\right\}=\left\{\frac{1}{2}+i b_{0}\right\}$ where $b_{0} \in\left(0, \frac{1}{2}\right)$ is the unique zero of the increasing function $e_{1}+\eta_{1}$ along $\frac{1}{2}+i b$. Then

$$
f\left(\frac{1}{2}+i b\right)=2\left(\frac{1}{2}+i b\right)-\frac{4 \pi i}{e_{1}+\eta_{1}}=1+\frac{2 b i}{e_{1}+\eta_{1}}\left(e_{1}+\eta_{1}-\frac{2 \pi}{b}\right) .
$$

In particular, $f\left(\frac{1}{2}+i b_{1}\right)=1, f\left(\frac{1}{2}+i b_{0}\right)=1-i \infty$. We recall the classical transformation law for $\eta_{1}$ :

$$
\eta_{1}\left(\tau^{\prime}\right)=(c \tau+d)^{2} \eta_{1}(\tau)-2 \pi i c(c \tau+d),
$$

for $\tau^{\prime}=\gamma \cdot \tau, \gamma \in \mathrm{SL}(2, \mathbb{Z})$. In particular, $\eta_{1}\left(\frac{1}{\tau}\right)=\tau^{2} \eta_{1}(\tau)-2 \pi i \tau$. From those transformation, we have $\eta_{1}(\tau)+e_{1}(\tau) \rightarrow \infty$ when $\tau \in C_{1}\left(F_{2}\right) \cup C_{1}\left(F_{2}^{\prime}\right)$ tends to the boundary point 0 (resp. 1 ), we have that $f(\tau) \rightarrow 0$ (resp. 2). Therefore $f$ maps $\overline{C_{1}\left(F_{0}\right)}$ and $\overline{C_{1}\left(F_{0}^{\prime}\right)}$ onto $[0,2]$ and $\mathbb{R} \cup\{\infty\} \backslash(0,2)$ respectively. Then $f(\tau)=\infty$ has only one solution in $F$ at $\tau=\frac{1}{2}+i b_{0}$.

Now let $G$ be any fundamental domain of $\Gamma(2)$ and $G=\gamma \cdot F$. Let $p$ and $q$ are the cusps corresponding to 0 and 1 by $\gamma$. It is easy to see that from the transformation $\eta_{1}(\gamma \cdot \tau)=(c \tau+d)^{2} \eta_{1}(\tau)-2 \pi i c(c \tau+d)$, we have $\eta_{1}(\tau)+e_{1}(\tau) \rightarrow \infty$ as $\tau \rightarrow p, q$. Thus this proves the theorem.

REMARK 6.10 . In the proof, we see that $f(\tau)$ maps $\bar{D}$ to $C_{-} \cup \mathbb{R}$ locally one-one. It is easy to see that the map is onto and globally one-one.

\section{A generalization of the Hitchin theorem}

7.1. The statement. In this section, we want to give a proof of Theorem 4.10. Recall the premodular form $Z_{r, s}^{(n)}(\tau)$ associated with the hyperelliptic curve $Y^{(n)}(\tau)$. For convenience, we use $Z_{r, s}^{(0)}(\tau)$ and $Z_{r, s}^{(1)}$ to denote 1 and $Z_{r, s}(\tau)$, respectively. Recall that in the last section, we use the Hitchin Theorem to obtain that $Z_{r, s}(\tau)$ satisfies the simple zeros property. The following theorem is a generalization of Hitchin theorem to Painlevé $\operatorname{VI}\left(\alpha_{i}\right)$, where

$$
\alpha_{0}=\frac{1}{2}\left(n+\frac{1}{2}\right)^{2} \text { and } \alpha_{i}=\frac{1}{8}, \quad 1 \leq i \leq 3 .
$$

Theorem 7.1. [9] There exists a polynomial $P_{n+1}(X ; \sigma) \in Q\left[g_{2}, g_{3}\right.$, $\left.\wp(\sigma), \wp^{\prime}(\sigma), X\right]$ such that for any $(r, s) \in \mathbb{C}^{2} \backslash \frac{1}{2} \mathbb{Z}^{2}$, the following $p_{r, s}^{(n)}(\tau)$ sat- 
isfies the Painlevé VI $\left(\alpha_{i}\right)$, where $\alpha_{i}$ is given by (7.1) and

$$
\wp\left(p_{r, s}^{(n)}(\tau)\right)=\wp(r+s \tau)+\frac{P_{n+1}\left(Z_{r, s}(\tau) ; r+s \tau\right)}{Z_{r, s}^{(n-1)}(\tau) Z_{r, s}^{(n+1)}(\tau)} .
$$

Furthermore, the followings holds:

(i) any two polynomials of $W^{(n-2)}(X ; \sigma), W^{(n)}(X ; \sigma)$ and $P_{n+1}(X ; \sigma)$ has no common roots for any $\sigma \notin E_{\tau}[2]$, where $W^{(n)}(\tau) \equiv 1$ if $n \leq 0$.

(ii) any two polynomials of $W^{(n)}(X ; \sigma), W^{(n+1)}(X, \sigma)$ and $W^{(n+2)}(X, \sigma)$ has no common roots for any $\sigma \notin E_{\tau}[2]$.

(iii) The monodromy group of the GLE associated with $p_{r, s}^{(n)}(\tau)$ is generated by $\left(\begin{array}{cc}e^{2 \pi i s} & 0 \\ 0 & e^{-2 \pi i s}\end{array}\right)$ and $\left(\begin{array}{cc}e^{-2 \pi i r} & 0 \\ 0 & e^{2 \pi i r}\end{array}\right)$. Conversely, any completely reducible solutions are given by (7.2).

Due to (i), the denominator and numerator of the RHS in (7.2) can not vanish simultaneously. So we have

Corollary 7.2. Suppose $Z_{r, s}^{(n)}\left(\tau_{0}\right)=0$, then $\tau_{0}$ is a simple zero of $Z_{r, s}^{(n)}(\tau)$ if $(r, s) \in \mathbb{C} \backslash \frac{1}{2} \mathbb{Z}^{2}$ and $r+s \tau_{0} \notin E_{\tau_{0}}[2]$.

The proof of Theorem 7.1 is to apply the Okamoto transformation. The Okamoto transformation is a bi-rational transformation of the solutions of the Hamiltonian systems related to Painlevé VI $\left(\frac{1}{8}\right)$ and Painlevé VI $\left(\alpha_{i}\right)$ of (7.1). See $[23,25,31]$. The formula for this Okamoto transformation is very complicated. In the followings, we will briefly describe it.

7.2. Okamoto transformation. We recall that PVI is equivalent to the following Hamiltonian system

$$
\frac{d \lambda(t)}{d t}=\frac{\partial K}{\partial \mu}, \quad \frac{d \mu(t)}{d t}=-\frac{\partial K}{\partial \lambda},
$$

where $K=K(\lambda, \mu, t)$ is given by

$$
K=\frac{1}{t(t-1)}\left\{\begin{array}{l}
\lambda(\lambda-1)(\lambda-t) \mu^{2}+\theta_{0}\left(\theta_{0}+\theta_{4}\right)(\lambda-t) \\
-\left[\begin{array}{l}
\theta_{1}(\lambda-1)(\lambda-t)+\theta_{2} \lambda(\lambda-t) \\
+\left(\theta_{3}-1\right) \lambda(\lambda-1)
\end{array}\right] \mu
\end{array}\right\},
$$

and the relation of parameters is given by

$$
\begin{gathered}
(\alpha, \beta, \gamma, \delta)=\left(\frac{1}{2} \theta_{4}^{2},-\frac{1}{2} \theta_{1}^{2}, \frac{1}{2} \theta_{2}^{2}, \frac{1}{2}\left(1-\theta_{3}^{2}\right)\right), \\
\theta_{0}:=\frac{1}{2}\left(1-\theta_{1}-\theta_{2}-\theta_{3}-\theta_{4}\right), \\
\text { i.e., } \quad 2 \theta_{0}+\theta_{1}+\theta_{2}+\theta_{3}+\theta_{4}=1 .
\end{gathered}
$$

It is known that solutions of PVI with parameters

$$
\begin{aligned}
(\alpha, \beta, \gamma, \delta)= & \left(\frac{1}{2}\left(n_{0}+\frac{1}{2}\right)^{2},-\frac{1}{2}\left(n_{1}+\frac{1}{2}\right)^{2}, \frac{1}{2}\left(n_{2}+\frac{1}{2}\right)^{2},\right. \\
& \left.\frac{1}{2}-\frac{1}{2}\left(n_{3}+\frac{1}{2}\right)^{2}\right), n_{k} \in \mathbb{N} \cup\{0\} \text { for all } k .
\end{aligned}
$$


could be obtained from solutions of $\operatorname{PVI}\left(\frac{1}{8}, \frac{-1}{8}, \frac{1}{8}, \frac{3}{8}\right)$ (i.e., $n_{k}=0$ for all $k$ ) via the Okamoto transformation $([\mathbf{3 1}])$.

First we recall the explicit form of the Okamoto transformations. By (7.5) and (7.6), it is convenient to think of the parameter space of PVI (7.1) (equivalently the Hamiltonian system (7.3)-(7.4)) as an affine space

$$
\mathcal{K}=\left\{\theta=\left(\theta_{0}, \theta_{1}, \theta_{2}, \theta_{3}, \theta_{4}\right) \in \mathbb{C}^{5}: 2 \theta_{0}+\theta_{1}+\theta_{2}+\theta_{3}+\theta_{4}=1\right\} .
$$

An Okamoto transformation $\sigma$ maps solutions $(\lambda(t), \mu(t), t)$ of the Hamiltonian system (7.3) with parameter $\theta \in \mathcal{K}$ to solutions $\sigma(\lambda)(t)(\sigma(\lambda)(t)$, $\sigma(\mu)(t), t)$ of $(7.3))$ with new parameter $\sigma(\theta) \in \mathcal{K}$. The list of the Okamoto transformations $\sigma_{j}(0 \leq j \leq 4)$ is given in the Table 1 (cf. $[\mathbf{3 3}]$ ).

TABLE 1. Okamoto transformations

\begin{tabular}{|c|c|c|c|c|c|c|c|c|}
\hline & $\theta_{0}$ & $\theta_{1}$ & $\theta_{2}$ & $\theta_{3}$ & $\theta_{4}$ & $t$ & $\lambda$ & $\mu$ \\
\hline$\sigma_{0}$ & $-\theta_{0}$ & $\theta_{1}+\theta_{0}$ & $\theta_{2}+\theta_{0}$ & $\theta_{3}+\theta_{0}$ & $\theta_{4}+\theta_{0}$ & $t$ & $\lambda+\frac{\theta_{0}}{\mu}$ & $\mu$ \\
\hline$\sigma_{1}$ & $\theta_{0}+\theta_{1}$ & $-\theta_{1}$ & $\theta_{2}$ & $\theta_{3}$ & $\theta_{4}$ & $t$ & $\lambda$ & $\mu-\frac{\theta_{1}}{\lambda}$ \\
\hline$\sigma_{2}$ & $\theta_{0}+\theta_{2}$ & $\theta_{1}$ & $-\theta_{2}$ & $\theta_{3}$ & $\theta_{4}$ & $t$ & $\lambda$ & $\mu-\frac{\theta_{2}}{\lambda-1}$ \\
\hline$\sigma_{3}$ & $\theta_{0}+\theta_{3}$ & $\theta_{1}$ & $\theta_{2}$ & $-\theta_{3}$ & $\theta_{4}$ & $t$ & $\lambda$ & $\mu-\frac{\theta_{3}}{\lambda-t}$ \\
\hline$\sigma_{4}$ & $\theta_{0}+\theta_{4}$ & $\theta_{1}$ & $\theta_{2}$ & $\theta_{3}$ & $-\theta_{4}$ & $t$ & $\lambda$ & $\mu$ \\
\hline
\end{tabular}

For example, the $\theta$ parameter is $\left(-\frac{1}{2}, \frac{1}{2}, \frac{1}{2}, \frac{1}{2}, \frac{1}{2}\right)$ for the Hitchin equation and is $\left(\frac{1}{2}, 0,0,0,0\right)$ for the Picard equation (whose solutions are called Picard solutions). From Table 1 , we see $\sigma_{0}$ maps $\left(\frac{1}{2}, 0,0,0,0\right)$ to $\left(-\frac{1}{2}, \frac{1}{2}, \frac{1}{2}, \frac{1}{2}, \frac{1}{2}\right)$. Indeed the inverse transformation of $\sigma_{0}$ is given by

$$
\hat{\lambda}(t)=\lambda(t)-\frac{1}{2 \mu(t)}, \mu(t)=\frac{t(t-1) \lambda^{\prime}+\frac{1}{2} \lambda^{2}-t\left(\lambda-\frac{1}{2}\right)}{2 \lambda(\lambda-1)(\lambda-t)},
$$

where $(\lambda(t), \mu(t))$ is a solution of the Hamiltonian system corresponding to the Hitchin equation, and $\hat{\lambda}(t)$ is the corresponding solution of the Picard equation.

SeCOnd PROOF of THE Hitchin theOREm. As an application of the Okamoto transformation, we give a rigorous derivation of Picard solution from Hitchin's formula by the inverse transformation $\sigma_{0}$. First, we derive any Picard solution can be written as

$$
\hat{\lambda}(t)=\hat{\lambda}_{r, s}(t)=\frac{\wp(r+s \tau \mid \tau)-e_{1}(\tau)}{e_{2}(\tau)-e_{1}(\tau)}, \quad t=\frac{e_{3}(\tau)-e_{1}(\tau)}{e_{2}(\tau)-e_{1}(\tau)} .
$$

Recall the Picard solution is $p(\tau)=r+s \tau$. From (6.2), we have $\frac{t+1}{3}=$ $\frac{-e_{1}(\tau)}{e_{2}(\tau)-e_{1}(\tau)}$, and it is well known that locally the inverse function of $t(\tau)=$ $\frac{e_{3}(\tau)-e_{1}(\tau)}{e_{2}(\tau)-e_{1}(\tau)}$ can be expressed as

$$
\tau=\tau(t)=i \frac{F\left(\frac{1}{2}, \frac{1}{2}, 1 ; t\right)}{F\left(\frac{1}{2}, \frac{1}{2}, 1 ; 1-t\right)}=\frac{\omega_{2}(t)}{\omega_{1}(t)}
$$


where $F(\alpha, \beta, \gamma ; t)$ is the hypergeometric function. Furthermore,

$$
e_{2}(\tau)-e_{1}(\tau)=-\pi^{2} F\left(\frac{1}{2}, \frac{1}{2}, 1 ; 1-t\right)^{2}=\omega_{1}(t)^{2} .
$$

Therefore, we easily deduce from (6.2) that

$$
\hat{\lambda}(t)=\frac{\wp(r+s \tau \mid 1, \tau)}{\omega_{1}(t)^{2}}-\frac{e_{1}(\tau)}{e_{2}(\tau)-e_{1}(\tau)}=\frac{\wp(r+s \tau \mid \tau)-e_{1}(\tau)}{e_{2}(\tau)-e_{1}(\tau)},
$$

which proves (7.9).

On the other hand, the above expression of Picard solutions can be obtained from the inverse of $\sigma_{0}$ as follows. Let $\lambda(t)=\lambda_{r, s}(t)$ given by Hitchin's formula (6.18), namely

$$
\lambda(t)=\frac{\wp\left(p_{r, s}(\tau) \mid \tau\right)-e_{1}(\tau)}{e_{2}(\tau)-e_{1}(\tau)}=\frac{\wp(r+s \tau \mid \tau)-e_{1}(\tau)+\frac{\wp^{\prime}(r+s \tau \mid \tau)}{2 Z_{r, s}(\tau)}}{e_{2}(\tau)-e_{1}(\tau)},
$$

where $Z_{r, s}$ is the Hecke form. The important thing is that, by studying the isomonodromic deformation of GLE (5.25) with $n=0$, we proved in $[\mathbf{1 0}$, Theorem 4.7] (without computing $\lambda^{\prime}(t)$ ) that

$$
\begin{aligned}
\mu(t) & =\frac{e_{2}(\tau)-e_{1}(\tau)}{2\left(\wp\left(p_{r, s}(\tau) \mid \tau\right)-\wp(r+s \tau \mid \tau)\right)} \\
& =\frac{\left(e_{2}(\tau)-e_{1}(\tau)\right) Z_{r, s}(\tau)}{\wp^{\prime}(r+s \tau \mid \tau)} .
\end{aligned}
$$

We do not think that it is easy to obtain (7.11) via (7.10) and the second formula of (7.8). Substituting (7.10) and (7.11) into the first formula of (7.8), we immediately obtain the same formula (7.9) of Picard solution $\hat{\lambda}(t)$.

These five transformations $\sigma_{j}(0 \leq j \leq 4)$, which satisfy $\sigma_{j} \circ \sigma_{j}=I d$, generate the affine Weyl group of type $D_{4}^{(1)}$ :

$$
W\left(D_{4}^{(1)}\right)=\left\langle\sigma_{0}, \sigma_{1}, \sigma_{2}, \sigma_{3}, \sigma_{4}\right\rangle .
$$

In $W\left(D_{4}^{(1)}\right)$ there are four special transformations that interest to us very much. Define

$$
\begin{aligned}
\sigma_{5} & :=\sigma_{0}\left(\sigma_{3} \sigma_{2} \sigma_{1} \sigma_{0}\right)^{2} \sigma_{4}, \\
\sigma_{6} & :=\sigma_{0}\left(\sigma_{4} \sigma_{2} \sigma_{1} \sigma_{0}\right)^{2} \sigma_{3}, \\
\sigma_{7} & :=\sigma_{0}\left(\sigma_{4} \sigma_{3} \sigma_{1} \sigma_{0}\right)^{2} \sigma_{2}, \\
\sigma_{8} & :=\sigma_{0}\left(\sigma_{4} \sigma_{3} \sigma_{2} \sigma_{0}\right)^{2} \sigma_{1} .
\end{aligned}
$$

A straightforward computation shows

$$
\begin{aligned}
& \sigma_{5}(\theta)=\left(\theta_{0}-1, \theta_{1}, \theta_{2}, \theta_{3}, \theta_{4}+2\right), \\
& \sigma_{6}(\theta)=\left(\theta_{0}-1, \theta_{1}, \theta_{2}, \theta_{3}+2, \theta_{4}\right), \\
& \sigma_{7}(\theta)=\left(\theta_{0}-1, \theta_{1}, \theta_{2}+2, \theta_{3}, \theta_{4}\right), \\
& \sigma_{8}(\theta)=\left(\theta_{0}-1, \theta_{1}+2, \theta_{2}, \theta_{3}, \theta_{4}\right) .
\end{aligned}
$$


Then for any $n_{k} \in \mathbb{N} \cup\{0\}$ for all $k$, it is easy to see that $(7.15)$

$$
\left(\theta_{0}, \theta_{1}, \theta_{2}, \theta_{3}, \theta_{4}\right)=\left(-\frac{1+\sum_{k=0}^{3} n_{k}}{2}, n_{1}+\frac{1}{2}, n_{2}+\frac{1}{2}, n_{3}+\frac{1}{2}, n_{0}+\frac{1}{2}\right)
$$

can be obtained from $\left(-\frac{1}{2}, \frac{1}{2}, \frac{1}{2}, \frac{1}{2}, \frac{1}{2}\right)$ (which corresponds to $\operatorname{PVI}\left(\frac{1}{8}, \frac{-1}{8}, \frac{1}{8}, \frac{3}{8}\right)$ ) by using $\sigma_{j}$ with $1 \leq j \leq 8$. But for Theorem 7.1, we have $n_{1}=n_{2}=n_{3}=0$ and only need $\sigma_{5}$.

LEMma 7.3. [9] Let $(\lambda(t), \mu(t))$ be a solution of the Hamiltonian system (7.3) with parameter $\theta \in \mathcal{K}$. Then $(\tilde{\lambda}(t), \tilde{\mu}(t)) \doteqdot\left(\sigma_{5}(\lambda)(t), \sigma_{5}(\mu)(t)\right)$, which is a solution of the Hamiltonian system (7.3) with new parameter

$$
\tilde{\theta}=\left(\tilde{\theta}_{0}, \tilde{\theta}_{1}, \tilde{\theta}_{2}, \tilde{\theta}_{3}, \tilde{\theta}_{4}\right) \doteqdot \sigma_{5}(\theta)=\left(\theta_{0}-1, \theta_{1}, \theta_{2}, \theta_{3}, \theta_{4}+2\right),
$$

are expressed as

$$
\begin{aligned}
& \tilde{\lambda}(t)=\hat{\lambda}(t)+\frac{1-\theta_{0}}{\tilde{\mu}(t)}, \\
& \tilde{\mu}(t)=\hat{\mu}(t)+\frac{\theta_{0}+\theta_{1}-1}{\hat{\lambda}(t)}+\frac{\theta_{0}+\theta_{2}-1}{\hat{\lambda}(t)-1}+\frac{\theta_{0}+\theta_{3}-1}{\hat{\lambda}(t)-t},
\end{aligned}
$$

where

$$
\begin{aligned}
& \hat{\lambda}(t)=\bar{\lambda}(t)+\frac{1+\theta_{4}}{\hat{\mu}(t)}, \quad \bar{\lambda}(t)=\lambda(t)+\frac{\theta_{0}+\theta_{4}}{\mu(t)}, \\
& \hat{\mu}(t)=\mu(t)-\frac{\theta_{0}+\theta_{1}+\theta_{4}}{\bar{\lambda}(t)}-\frac{\theta_{0}+\theta_{2}+\theta_{4}}{\bar{\lambda}(t)-1}-\frac{\theta_{0}+\theta_{3}+\theta_{4}}{\bar{\lambda}(t)-t} .
\end{aligned}
$$

Proof. The proof is just a straightforward computation via Table 1.

Now we focus on the corresponding parameter $\theta^{n}$ by

$$
\begin{gathered}
\theta^{n} \doteqdot\left(-\frac{n+1}{2}, \frac{1}{2}, \frac{1}{2}, \frac{1}{2}, n+\frac{1}{2}\right), n \in \mathbb{N} \cup\{0\}, \\
\text { i.e., }(\alpha, \beta, \gamma, \delta)=\left(\frac{1}{2}\left(n+\frac{1}{2}\right)^{2},-\frac{1}{8}, \frac{1}{8}, \frac{3}{8}\right), n \in \mathbb{N} \cup\{0\} .
\end{gathered}
$$

Let $\sigma^{n-1, n} \in W\left(D_{4}^{(1)}\right), n \geq 1$, be any Okamoto transformation satisfying $\sigma^{n-1, n}\left(\theta^{n-1}\right)=\theta^{n}$. Recall that the Hitchin theorem shows that any completely reducible solution $p(\tau)$ of $\operatorname{PVI}\left(\frac{1}{8}\right)$ is given by

$$
\wp(p(\tau) \mid \tau)=\wp(r+s \tau \mid \tau)+\frac{\left.\wp^{\prime}(r+s \tau) \mid \tau\right)}{2 Z_{r, s}(\tau)}
$$

with $(r, s) \in \mathbb{C}^{2} \backslash \frac{1}{2} \mathbb{Z}^{2}$. Then by the transformation (6.2) and the Hamiltonian system (6.11), we have corresponding solution of the Hamiltonian system (6.11), denoted by $\left(\lambda_{r, s}^{0}(t), \mu_{r, s}^{0}(t)\right)$. By induction, for any $(r, s) \in \mathbb{C}^{2} \backslash \frac{1}{2} \mathbb{Z}^{2}$, we denote the corresponding completely reducible solution to PVI with parameter $\theta^{n}$ (i.e., $(7.20)$ and $\left.(7.21)\right)$ by $\lambda_{r, s}^{(n)}(t)$ and $p_{r, s}^{(n)}(\tau)$, that is,

$$
\left(\lambda_{r, s}^{(n)}(t), \mu_{r, s}^{(n)}(t)\right)=\sigma^{n-1, n}\left(\lambda_{r, s}^{(n-1)}(t), \mu_{r, s}^{(n-1)}(t)\right) .
$$


In the following, we usually omit the subscripts $r, s$ for convenience. The following result gives the explicit expression of $\left(\lambda^{(n)}(t), \mu^{(n)}(t)\right)$ in terms of $\left(\lambda^{(n-1)}(t), \mu^{(n-1)}(t)\right)$.

LEMMA 7.4. [9] Under the above notations, for $n \geq 1$ there holds:

$$
\begin{aligned}
\mu^{(n)}= & \mu^{(n-1)}-\frac{n}{2}\left(\frac{1}{\lambda^{(n-1)}+\frac{n-1}{2 \mu^{(n-1)}}}\right. \\
& \left.+\frac{1}{\lambda^{(n-1)}+\frac{n-1}{2 \mu^{(n-1)}}-1}+\frac{1}{\lambda^{(n-1)}+\frac{n-1}{2 \mu^{(n-1)}}-t}\right), \\
\lambda^{(n)}= & \lambda^{(n-1)}+\frac{n-1}{2 \mu^{(n-1)}}+\frac{n+1}{2 \mu^{(n)}} .
\end{aligned}
$$

We prove Theorem 7.1 by induction on $n$. Here (7.22) and (7.23) are our formula for the induction process. Nevertheless, the proof still contains many complicate compilations. See $[\mathbf{9}]$ for the complete proof.

We remark that in general the right hand side of 7.2 is very difficult to compute either by the method in [27] or the so-called Krichever's Ansatz. Our proof suggests the Okamoto transformation could be useful in such a computation. In the followings, we provide some examples of (7.2) by applying Okamoto transformation.

EXAMPLE 7.5. $a=a(\tau)=r+s \tau, Z_{r, s}^{(1)}(\tau)=\zeta(r+s \tau)-r \eta_{1}(\tau)-s \eta_{2}(\tau)$.

(i) For $n=2$, we have

$$
\begin{aligned}
Z_{r, s}^{(2)}(\tau) & =k^{3}-3 \wp(a) k-\wp^{\prime}(a) \\
\wp\left(p_{r, s}^{(1)}(\tau)\right) & =\wp(r+s \tau)+\frac{3 \wp^{\prime}(a) k^{2}+\left(12 \wp(a)^{2}-g_{2}\right) k+3 \wp(a) \wp^{\prime}(a)}{2 Z_{r, s}^{(2)}(\tau)} .
\end{aligned}
$$

(ii) For $n=3$, we have

$$
\begin{aligned}
Z_{r, s}^{(3)}(\tau ; \sigma)= & k^{6}-15 \wp(\sigma) k^{4}-20 \wp^{\prime}(\sigma) k^{3}+\left(\frac{27}{4} g_{2}-15 \wp(\sigma)^{2}\right) k^{2} \\
& -12 \wp(\sigma) \wp^{\prime}(\sigma) k-\frac{5}{4} \wp^{\prime}(\sigma)^{2}
\end{aligned}
$$

$$
\begin{aligned}
& \wp\left(p_{r, s}^{(2)}(\tau)\right)=\wp(r+s \tau) \\
& +\frac{28 \wp^{\prime}(\sigma) k^{6}+\left(288 \wp(\sigma)^{2}-24 g_{2}\right) k^{5}+300 \wp(\sigma) \wp^{\prime}(\sigma) k^{4}+1640 \wp(\sigma)^{3}-88 g_{2} \wp(\sigma)}{8 Z_{r, s}^{(1)}(\tau ; \sigma) Z_{r, s}^{(3)}(\tau ; \sigma)},
\end{aligned}
$$

where $\sigma=r+s \tau$.

In the next subsection, we will discuss the Okamoto transformation for not completely reducible solutions and its application. For the simplicity, we only consider the case $n=2$. 
7.3. Smoothness of degenerate curves for $n=2$. Now we come to compute the Hessian of $G^{(n)}(z)$ on $E_{\tau}^{n}$ at each branch point of $Y^{(n)}(\tau)$

$$
G^{(n)}(z)=n \sum_{i=1}^{n} G\left(z_{i}\right)-\sum_{i<j} G\left(z_{i}-z_{j}\right), \quad z=\left(z_{1}, \ldots, z_{n}\right) .
$$

The calculation is highly non-trivial even for $n=2$. Here we present the case $n=2$ in order to show how it is related to Painlevé VI.

In section 3 , we have shown that $Y^{(2)}(\tau)$ has 5 branch points: $\left(\frac{\omega_{i}}{2}, \frac{\omega_{j}}{2}\right)$ and $\left(q_{ \pm},-q_{ \pm}\right)$, where $\wp\left(q_{ \pm}\right)= \pm \sqrt{\frac{g_{2}}{12}}$. The Hessian at those points are

$$
\operatorname{det}\left(D^{2} G^{(2)}\left(\frac{\omega_{i}}{2}, \frac{\omega_{j}}{2}\right)\right)=\frac{4\left|3 e_{k} \eta_{1}+\frac{g_{2}}{2}-3 e_{k}^{2}\right|}{(2 \pi)^{4} \operatorname{Im} \tau} \operatorname{Im}\left(\tau-\frac{6 \pi i}{3 e_{k} \eta_{1}+\frac{g_{2}}{2}-3 e_{k}^{2}}\right),
$$

and

$$
\operatorname{det}\left(D^{2} G^{(2)}\left(q_{ \pm},-q_{ \pm}\right)\right)=\frac{3 g_{2}}{4 \pi^{4} \operatorname{Im} \tau}\left|\wp\left(q_{ \pm}\right)+\eta_{1}\right|^{2} \operatorname{Im}\left(\tau-\frac{2 \pi i}{\wp\left(q_{ \pm}\right)+\eta_{1}}\right) .
$$

We let $C_{i j}$ or $C_{ \pm}$denote the curve of $\tau$ such that $\left(\frac{\omega_{i}}{2}, \frac{\omega_{j}}{2}\right)$ or $\left(q_{ \pm},-q_{ \pm}\right)$are degenerate critical points of $G^{(2)}(\tau)$.

Theorem 3.6 asserts that $C_{i j}$ or $C_{ \pm}$has no self-intersections. This is equivalent to the following result.

THEOREM 7.6. The meromorphic functions

$$
\begin{aligned}
& \phi_{ \pm}(\tau):=\tau-\frac{2 \pi i}{\eta_{1}(\tau) \pm \sqrt{\frac{g_{2}(\tau)}{12}}}, \text { and } \\
& \phi_{k}(\tau):=\tau-\frac{6 \pi i e_{k}(\tau)}{3 e_{k}(\tau) \eta_{1}(\tau)+\frac{g_{2}(\tau)}{2}-3 e_{k}(\tau)^{2}} .
\end{aligned}
$$

are locally 1-1.

To prove Theorem 7.6, we have to express the non-completely reducible solutions of Painlevé $\left(\alpha_{i}\right), \alpha=\left(\frac{9}{8}, \frac{1}{8}, \frac{1}{8}, \frac{1}{8}\right)$.

THEOREM 7.7. Suppose that $p^{(1)}(\tau)$ is a solution to the elliptic form

$$
\frac{d^{2} p(\tau)}{d \tau^{2}}=\frac{-1}{32 \pi^{2}} \sum_{i=1}^{3} \wp^{\prime}\left(p(\tau)+\frac{\omega_{i}}{2} \mid \tau\right)+\frac{-9}{32 \pi^{2}} \wp^{\prime}(p(\tau) \mid \tau) .
$$

Then $p^{(1)}(\tau)$ is a non completely reducible solution if and only if there exists constant $C \in \mathbb{C} \cup\{\infty\}$ such that either

$$
\begin{aligned}
& \wp\left(p^{(1)}(\tau) \mid \tau\right) \\
= & \frac{-4\left(C \eta_{1}-\eta_{2}\right)^{3}-g_{2}\left(C \eta_{1}-\eta_{2}\right)(C-\tau)^{2}+2 g_{3}(C-\tau)^{3}}{(C-\tau)\left[12\left(C \eta_{1}-\eta_{2}\right)^{2}-g_{2}(C-\tau)^{2}\right]},
\end{aligned}
$$


or

$$
\wp\left(p^{(1)}(\tau) \mid \tau\right)=\frac{\left(\frac{g_{2}}{2}-3 e_{k}^{2}\right)\left(C \eta_{1}-\eta_{2}\right)+\frac{g_{2}}{4} e_{k}(C-\tau)}{3 e_{k}\left(C \eta_{1}-\eta_{2}\right)+\left(\frac{g_{2}}{2}-3 e_{k}^{2}\right)(C-\tau)}
$$

with $k \in\{1,2,3\}$.

Remark that if $C=\infty$, then formulae (7.25)-(7.26) turn to be

$$
\begin{gathered}
\wp\left(p^{(1)}(\tau) \mid \tau\right)=\frac{-4 \eta_{1}^{3}-g_{2} \eta_{1}+2 g_{3}}{12 \eta_{1}^{2}-g_{2}}, \\
\wp\left(p^{(1)}(\tau) \mid \tau\right)=\frac{\left(\frac{g_{2}}{2}-3 e_{k}^{2}\right) \eta_{1}+\frac{g_{2}}{4} e_{k}}{3 e_{k} \eta_{1}+\frac{g_{2}}{2}-3 e_{k}^{2}},
\end{gathered}
$$

respectively.

For $C \in \mathbb{C} \cup\{\infty\}$ and $k \in\{1,2,3\}$, we define functions from $\mathbb{H}$ to $\mathbb{C} \cup\{\infty\}:$

$$
\begin{gathered}
f_{C}(\tau) \doteqdot\left\{\begin{array}{c}
12\left(C \eta_{1}(\tau)-\eta_{2}(\tau)\right)^{2}-g_{2}(\tau)(C-\tau)^{2} \text { if } C \neq \infty, \\
12 \eta_{1}(\tau)^{2}-g_{2}(\tau) \quad \text { if } \quad C=\infty,
\end{array}\right. \\
f_{k, C}(\tau) \doteqdot\left\{\begin{array}{l}
3 e_{k}(\tau)\left(C \eta_{1}(\tau)-\eta_{2}(\tau)\right) \\
+\left(\frac{g_{2}(\tau)}{2}-3 e_{k}(\tau)^{2}\right)(C-\tau) \text { if } C \neq \infty, \\
3 e_{k}(\tau) \eta_{1}(\tau)+\frac{g_{2}(\tau)}{2}-3 e_{k}(\tau)^{2} \quad \text { if } C=\infty,
\end{array}\right.
\end{gathered}
$$

$$
\begin{gathered}
\phi_{ \pm}(\tau) \doteqdot \tau-\frac{2 \pi i}{\eta_{1}(\tau) \pm \sqrt{g_{2}(\tau) / 12}} \\
\phi_{k}(\tau) \doteqdot \tau-\frac{6 \pi i e_{k}(\tau)}{3 e_{k}(\tau) \eta_{1}(\tau)+\frac{g_{2}(\tau)}{2}-3 e_{k}(\tau)^{2}}=\tau-\frac{6 \pi i e_{k}(\tau)}{f_{k, \infty}(\tau)} .
\end{gathered}
$$

Notice that $\phi_{ \pm}(\tau)$ have branch points at $\tau \in \mathfrak{S}$, where

$$
\mathfrak{S} \doteqdot\left\{\frac{a e^{\pi i / 3}+b}{c e^{\pi i / 3}+d} \mid\left(\begin{array}{ll}
a & b \\
c & d
\end{array}\right) \in S L(2, \mathbb{Z})\right\},
$$

because $g_{2}(\tau)=0$ if and only if $\tau \in \mathfrak{S}$. Remark that

$$
\phi_{ \pm}(\tau)=\tau-\frac{2 \pi i}{\eta_{1}(\tau)}=\frac{\eta_{2}(\tau)}{\eta_{1}(\tau)} \notin \mathbb{R} \cup\{\infty\}, \quad \forall \tau \in \mathfrak{S} .
$$

TheOREM 7.8. For $C \in \mathbb{C P}^{1}$ and $k \in\{1,2,3\}$, let $f_{C}(\tau), f_{k, C}(\tau), \phi_{ \pm}(\tau)$ and $\phi_{k}(\tau)$ be defined in (7.27)-(7.30).

(i) Any of $f_{C}(\tau)$ and $f_{k, C}(\tau)$ has simple zeros only in $\mathbb{H}$.

(ii) $\phi_{ \pm}^{\prime}(\tau) \neq 0$ for any $\tau \in \mathbb{H} \backslash \mathfrak{S}$. In particular, $\phi_{ \pm}(\tau)$ has only simple zeros in $\mathbb{H}$ and is locally one-to-one from $\mathbb{H} \backslash \mathfrak{S}$ to $\mathbb{C} \cup\{\infty\}$.

(iii) For $k \in\{1,2,3\}, \phi_{k}^{\prime}(\tau) \neq 0$ for any $\tau \in \mathbb{H}$. In particular, $\phi_{k}(\tau)$ has only simple zeros in $\mathbb{H}$ and is locally one-to-one from $\mathbb{H}$ to $\mathbb{C} \cup\{\infty\}$.

Theorem 7.6 is a direct consequence of Theorem 7.8 . 
Proof. (i) Recalling

$$
\lambda^{(1)}(t)=\frac{\wp\left(p^{(1)}(\tau) \mid \tau\right)-e_{1}(\tau)}{e_{2}(\tau)-e_{1}(\tau)}, \quad t=t(\tau)=\frac{e_{3}(\tau)-e_{1}(\tau)}{e_{2}(\tau)-e_{1}(\tau)},
$$

Theorem 7.7 imply that for any zero $\tau_{0} \in \mathbb{H}$ of $f_{C}(\tau)$ or $f_{k, C}(\tau), t\left(\tau_{0}\right) \in$ $\mathbb{C} \backslash\{0,1\}$ is a pole of the corresponding solution $\lambda^{(1)}(t)$ to $\operatorname{PVI}\left(\frac{9}{8}, \frac{-1}{8}, \frac{1}{8}, \frac{3}{8}\right)$. Since $t^{\prime}(\tau) \neq 0$ for all $\tau \in \mathbb{H}$ and any pole $t_{0} \in \mathbb{C} \backslash\{0,1\}$ of $\lambda^{(1)}(t)$ is a simple pole, the assertion (i) follows readily.

(ii) Consider function $\phi_{+}(\tau)$. Fix any $\tau_{0} \in \mathbb{H} \backslash \mathfrak{S}$, then $g_{2}\left(\tau_{0}\right) \neq 0$. If $\eta_{1}\left(\tau_{0}\right)+\sqrt{g_{2}\left(\tau_{0}\right) / 12}=0$, then $\tau_{0}$ is a simple zero of $f_{\infty}(\tau)$ of $(7.27)$. Thus $\phi_{+}(\tau)$ is locally 1-1 near $\tau_{0}$. It suffices to consider $\eta_{1}\left(\tau_{0}\right)+\sqrt{g_{2}\left(\tau_{0}\right) / 12} \neq 0$. Then by letting

$$
C \doteqdot \phi_{+}\left(\tau_{0}\right)=\tau_{0}-\frac{2 \pi i}{\eta_{1}\left(\tau_{0}\right)+\sqrt{g_{2}\left(\tau_{0}\right) / 12}},
$$

we see from $\tau \eta_{1}-\eta_{2}=2 \pi i$ that $\tau_{0}$ is a zero of

$$
\begin{aligned}
\psi(\tau) & \doteqdot 2 \pi i+(C-\tau)\left(\eta_{1}(\tau)+\sqrt{g_{2} / 12}\right) \\
& =C \eta_{1}(\tau)-\eta_{2}(\tau)+\sqrt{g_{2} / 12}(C-\tau),
\end{aligned}
$$

which implies that $\tau_{0}$ is a zero of

$$
f_{C}(\tau)=12\left[C \eta_{1}(\tau)-\eta_{2}(\tau)-\sqrt{g_{2} / 12}(C-\tau)\right] \psi(\tau) .
$$

Then (i) gives $\psi^{\prime}\left(\tau_{0}\right) \neq 0$, i.e.,

$$
\eta_{1}\left(\tau_{0}\right)+\sqrt{g_{2}\left(\tau_{0}\right) / 12}+\left.\left(\tau_{0}-C\right) \frac{d}{d \tau}\left(\eta_{1}(\tau)+\sqrt{g_{2} / 12}\right)\right|_{\tau=\tau_{0}} \neq 0
$$

Together with $(7.32)$ and (7.29), we easily obtain $\phi_{+}^{\prime}\left(\tau_{0}\right) \neq 0$. Similarly we can prove $\phi_{-}^{\prime}(\tau) \neq 0$ for any $\tau \in \mathbb{H} \backslash \mathfrak{S}$. This proves (ii).

(iii) Now we consider function $\phi_{k}(\tau)$ defined in (7.30). Fix $k \in\{1,2,3\}$ and any $\tau_{0} \in \mathbb{H}$. If $f_{k, \infty}\left(\tau_{0}\right)=0$, then it is easy to see that $e_{k}\left(\tau_{0}\right) \neq 0$ and so $\left.\frac{d}{d \tau}\left(\frac{e_{k}(\tau)}{f_{k, \infty}(\tau)}\right)\right|_{\tau=\tau_{0}}=\infty$, i.e., $\phi_{k}^{\prime}\left(\tau_{0}\right) \neq 0$. It suffices to consider $f_{k, \infty}\left(\tau_{0}\right) \neq 0$. Then by letting

$$
C \doteqdot \phi_{k}\left(\tau_{0}\right)=\tau_{0}-\frac{6 \pi i e_{k}\left(\tau_{0}\right)}{f_{k, \infty}\left(\tau_{0}\right)},
$$

it follows from $\tau \eta_{1}-\eta_{2}=2 \pi i$ that $f_{k, C}\left(\tau_{0}\right)=0$, where $f_{k, C}(\tau)$ is defined in (7.28), i.e.,

$$
f_{k, C}(\tau)=6 \pi i e_{k}(\tau)+(C-\tau) f_{k, \infty}(\tau) .
$$

Again (i) gives $f_{k, C}^{\prime}\left(\tau_{0}\right) \neq 0$, i.e.,

$$
6 \pi i e_{k}^{\prime}\left(\tau_{0}\right)-f_{k, \infty}\left(\tau_{0}\right)+\left(C-\tau_{0}\right) f_{k, \infty}^{\prime}\left(\tau_{0}\right) \neq 0 .
$$

Together with (7.33) and (7.30), we easily obtain $\phi_{k}^{\prime}\left(\tau_{0}\right) \neq 0$. This completes the proof. 


\section{Application}

8.1. Zeros of Eisenstein series of weight 1. In this section, we discuss the zeros of the Eisenstein series of weight 1, as an application of Hitchin theorem. Consider

$$
Z_{(N)}(\tau)=\prod_{(r, s) \in Q_{N}} Z_{r, s}(\tau)
$$

where $Q_{N}$ is the set of $N$-torsion. $Z_{(N)}(\tau)$ is a modular form of weight $\left|Q_{N}\right|$ w.r.t $\operatorname{SL}(2, \mathbb{Z})$. It is easy to write

$$
Z_{(N)}(\tau)=C_{N} \Delta(\tau)^{m}\left(\ell_{1, N}(j)\right)^{2}
$$

for some monic polynomial $\ell_{1, N}$ of $j$ and nonzero constant $C_{N}$, where $j(z)$ is the invariant function under $\operatorname{SL}(2, \mathbb{Z})$ and $m \in \mathbb{N}$.

TheOREM 8.1. [10] For any $N \geq 5$ with $N \neq 6$, the monic polynomial $\ell_{1, N}(j)$ determined by (8.1) has rational coefficients and satisfies

(i) for any zero $j_{0}$ of $\ell_{1, N}(j)$, there is an algebraic solution $\lambda_{r, s}(t),(r, s) \in$ $Q_{N}$, such that $j_{0}=j\left(\tau_{0}\right)$, where $t_{0}=t\left(\tau_{0}\right)$ satisfies $\lambda_{r, s}\left(t_{0}\right)=\infty$. Conversely, for any algebraic solution $\lambda_{r, s}(t),(r, s) \in Q_{N}$, if $\lambda_{r, s}\left(t_{0}\right)=$ $\infty$ for some $t_{0}=t\left(\tau_{0}\right)$, then $j_{0}=j\left(\tau_{0}\right)$ is a zero of $\ell_{1, N}(j)$.

(ii) $\ell_{1, N}(j)$ has distinct roots.

(iii) for any $N_{1} \neq N_{2}, \ell_{1, N_{1}}(j)$ and $\ell_{1, N_{2}}(j)$ have no common zeros.

(iv)

$$
\operatorname{deg} \ell_{1, N}=\left\{\begin{array}{l}
\frac{\left|Q_{N}\right|}{24} \text { if } N \text { is odd } \\
\frac{|Q N|}{24}-\frac{1}{2} \varphi\left(\frac{N}{2}\right) \text { if } N \text { is even }
\end{array}\right.
$$

where $\varphi(\cdot)$ is the Euler function.

Recall the elementary formulae

$$
\left|Q_{N}\right|=N^{2} \prod_{p \mid N, p \text { prime }}\left(1-\frac{1}{p^{2}}\right), \varphi(N)=N \prod_{p \mid N, p \text { prime }}\left(1-\frac{1}{p}\right) .
$$

Define

$$
\begin{aligned}
& J_{N}^{-}=\left\{(r, s) \in Q_{N} \mid 2 r+s=1 \text { and } \frac{1}{3}<s<\frac{1}{2}\right\}, \\
& J_{N}^{+}=\left\{(r, s) \in Q_{N} \mid 2 r+s=1 \text { and } 0<s<\frac{1}{3}\right\} .
\end{aligned}
$$

Then we have the following interesting result.

ThEOREM 8.2. For any $N \geq 5$ with $N \neq 6, \ell_{N}(j)$ has exactly $\# J_{N}^{+}$ real zeros in $(0,1728)$ and exactly $\# J_{N}^{-}$real zeros in $(-\infty, 0)$. Furthermore, $\ell_{N}(j)$ has no zeros in $\{0\} \cup[1728,+\infty)$.

It is very difficult to compute the polynomial $\ell_{1, N}(j)$ even for small $N$. To simplify the computation, we use a summation of solutions of the Painlevé 
VI, instead of the product $Z_{(N)}$ as follows. Fix $N \geq 5$ with $N \neq 6$. Define $J(N)$ to be the zero set of $\ell_{1, N}(j)$ :

$$
J(N):=\left\{j(\tau) \mid Z_{r, s}(\tau)=0 \text { for some }(r, s) \in Q_{N}\right\}=\left\{j_{k} \mid 1 \leq k \leq \operatorname{deg} \ell_{1, N}\right\} .
$$

Recall that $\lambda_{r, s}(t)$ is a solution of Painlevé VI $\left(\frac{1}{8},-\frac{1}{8}, \frac{1}{8}, \frac{3}{8}\right)$. Instead of considering the product like $Z_{(N)}(\tau)$, we consider the summation of $\lambda_{r, s}(t)$ with $(r, s) \in Q_{N}^{\prime}$ :

$$
y_{N}(t):=\sum_{(r, s) \in Q_{N}^{\prime}} \lambda_{r, s}(t)=\frac{1}{2} \sum_{(r, s) \in Q_{N}} \lambda_{r, s}(t)
$$

where

$$
Q_{N}^{\prime}=\left\{\begin{array}{l|l}
(r, s) \in Q_{N} & \begin{array}{l}
r<\frac{1}{2} \text { if } s=0 \\
s<\frac{1}{2} \text { if } r=0 \\
s \leq \frac{1}{2} \text { if } r \neq 0, s \neq 0
\end{array}
\end{array}\right\}
$$

Obviously, $y_{N}(t)$ is a rational function.

For $j_{k} \in J(N)$, since $j_{k} \notin\{0,1728\}$ by Theorem 8.2 , there are exactly six different $t$ 's which satisfies $j(t)=j_{k}$. We fix a $t_{k} \in \mathbb{C}$ such that $j\left(t_{k}\right)=j_{k}$, then $\Xi\left(t_{k}\right)$ gives precisely these six different $t$ 's. Therefore, we conclude that

$$
\bigcup_{k=1}^{\operatorname{deg} \ell_{1, N}} \Xi\left(t_{k}\right)=\bigcup_{k=1}^{\operatorname{deg} \ell_{1, N}}\left\{t_{k}, 1-t_{k}, \frac{1}{t_{k}}, 1-\frac{1}{t_{k}}, \frac{1}{1-t_{k}}, \frac{t_{k}}{t_{k}-1}\right\}
$$

gives precisely all the poles of $y_{N}(t)$.

From the above argument, we have

$$
y_{N}(t)=-\sum_{k=1}^{\operatorname{deg} \ell_{1, N}} \sum_{a \in \Xi\left(t_{k}\right)} \frac{2 a(a-1)}{t-a}+C t+D,
$$

where $C, D$ are two constants that can be easily determined.

We go back to the problem of computing $J(N)$ (or $\left.\ell_{1, N}(j)\right)$. The key observation is that the coefficients of the Taylor expression of $y_{N}(t)$ at $t=0$ are expressed in terms of $j_{k} \in J(N)$. In general, $J(N)$ can be determined by $y_{N}^{(2 L)}(0)$, the $2 L$-th derivatives, with $1 \leq L \leq \operatorname{deg} \ell_{1, N}$. On the other hand, we can compute the Taylor expansion of $y_{N}(t)$ at $t=0$ up to the term $t^{2 \operatorname{deg} \ell_{N}}$ by using Mathematica. In this way $J(N)$ can be computed. Here are some examples:

$$
J(3)=\{0\}, J(5)=\left\{\frac{5 \cdot 2^{12}}{3^{5}}\right\}, J(8)=\left\{\frac{207646}{3^{8}}\right\} .
$$

$N=7$, we have

$$
\ell_{1,7}=j^{2}+\frac{2^{12} \cdot 37001}{3^{2} \cdot 5^{7}} j-\frac{2^{24} \cdot 571787}{3^{7} \cdot 5^{7}}
$$

and

$$
J(7)=\left\{\frac{2^{11}}{5^{7} \cdot 3^{4}}(-333009 \pm 175519 \sqrt{21})\right\}
$$


For $N=9$, the polynomial is

$$
\begin{array}{r}
\ell_{1,9}(j)=j^{3}+\frac{86191391040000000}{78815638671875} j^{2}+\frac{19885648112869441536}{78815638671875} j \\
-\frac{7205712225604271603712}{78815638671875} .
\end{array}
$$

So $J(9)=\{a, b, \bar{b}\}$, where $a \in \mathbb{R}$ and $b \notin \mathbb{R}$. Numerically,

$$
a \approx 186.3, b \approx-639.9+285.0 \sqrt{-1} .
$$

It seems that except for $N=3$, all elements in $J(N)$ are not algebraic integers.

We ask the following question: Is any real root $j$ of $\ell_{1, N}, N \geq 5$, not an algebraic integer? The affirmative answer implies the corresponding $\tau$ is transcendental by a classical result of Schneider.

8.2. The proof of Dahmen-Beukers' conjecture. The second application is the proof of the conjecture of Dahmen-Beukers. To prove Dahmen-Beukers conjecture, we define

$$
M_{n, N}(\tau):=\prod_{(r, s) \in \mathcal{Q}(N)} Z_{r, s}^{(n)}(\tau) .
$$

Clearly $M_{n, N}(\tau)$ is a modular form of weight $\frac{n(n+1)}{2} \Psi(N)$ with respect to $S L(2, \mathbb{Z})$, where $\Psi(N)$ is given by (5.19). Set

$$
U_{n}(N)=\frac{1}{2}\left(\frac{n(n+1) \Psi(N)}{24}-v_{\infty}\left(M_{n, N}(\tau)\right)\right)+\frac{2}{3} \varepsilon_{n}(N),
$$

where $v_{\infty}\left(M_{n, N}(\tau)\right)$ denotes the vanishing order of $M_{n, N}(\tau)$ at infinity. Then the crucial result in both Dahmen's and Chou's proof of the conjecture for $n \in\{1,2,3,4\}$ is

Lemma 8.3. [4] There holds $L_{n}(N) \leq U_{n}(N)$. Furthermore, $L_{n}(N)=$ $U_{n}(N)$ if and only if $Z_{r, s}^{(n)}(\tau)$ has only simple zeros in $\mathbb{H}(r, s) \in \mathcal{Q}_{N}$.

See [4, Lemma 65] for the proof. For $n \in\{1,2,3,4\}$, the explicit expressions of $Z_{r, s}^{(n)}(\tau)$ are known; for example, see $\S 7$. Hence the vanishing order $v_{\infty}\left(M_{n, N}(\tau)\right)$ of $M_{n, N}(\tau)$ at $\tau=\infty$ can be calculated explicitly:

$$
v_{\infty}\left(M_{n, N}(\tau)\right)=a_{n} \phi(N)+b_{n} \phi\left(\frac{N}{2}\right)
$$

for $n \in\{1,2,3,4\}$, where $a_{n}$ and $b_{n}$ are given by (5.23). Then Lemma 8.3 yields

$$
\begin{aligned}
L_{n}(N) & \leq U_{n}(N) \\
& =\frac{1}{2}\left(\frac{n(n+1) \Psi(N)}{24}-\left(a_{n} \phi(N)+b_{n} \phi\left(\frac{N}{2}\right)\right)\right)+\frac{2}{3} \varepsilon_{n}(N) .
\end{aligned}
$$

This, together with (5.17) and (5.21), easily implies that for odd $N$,

$$
P L_{n}(N)=L_{n}(N)+L_{n}(2 N) \leq U_{n}(N)+U_{n}(2 N)=P L_{n}(N),
$$


so $L_{n}(N)=U_{n}(N)$ and $L_{n}(2 N)=U_{n}(2 N)$ if $N$ is odd. If $4 \mid N$ then $L_{n}(N)=$ $U_{n}(N)$ follows directly from (5.17) and (5.21). This proves Dahmen-Beukers conjecture for $n \in\{1,2,3,4\}$. A consequence of this proof and Lemma 8.3 is that for $n \in\{1,2,3,4\}, Z_{r, s}^{(n)}(\tau)$ has at most simple zeros in $\mathbb{H}$ if $(r, s) \in \mathcal{Q}_{N}$.

From the discussion above, the identity (8.8) is a crucial step to confirm Dahmen-Beukers conjecture. Our second main result is

Theorem 8.4. [9] For any $n \in \mathbb{N}$ and $N \in \mathbb{N}_{\geq 3}, v_{\infty}\left(M_{n, N}(\tau)\right)=$ $a_{n} \phi(N)+b_{n} \phi(N / 2)$ holds. Consequently, Dahmen-Beukers conjecture holds true.

REMARK 8.5. As pointed out above, in both Dahmen's and Chou's proof of the conjecture for $n \in\{1,2,3,4\}$, since the explicit expression of $Z_{r, s}^{(n)}(\tau)$ is known, they could prove the identity (8.8) first and then obtained the simple zero property of $Z_{r, s}^{(n)}(\tau)$ as a byproduct. However, this approach can not apply for general $n \geq 5$ because it is impossible to write down the explicit expression of $Z_{r, s}^{(n)}(\tau)$ for general $n \geq 5$. In this paper, we exploit an opposite idea, namely we prove the simple zero property of $Z_{r, s}^{(n)}(\tau)$ (i.e. Corollary 7.2) first, and then apply it to prove the identity (8.8)! Indeed, we need to prove that pre-modular forms $Z_{r, s}^{(n)}(\tau)$ possess the following properties:

(P-1) For any $N \in \mathbb{N}_{\geq 3}$ and $(r, s) \in \mathcal{Q}(N), Z_{r, s}^{(n)}(\tau)$ has only simple zeros in $\mathbb{H}$;

(P-2) For any $N \in \mathbb{N}_{\geq 3}, v_{\infty}\left(Z_{r, s}^{(n)}(\tau)\right)=0$ for any $(r, s) \in \mathcal{Q}(N)$ with $s \notin\left\{0, \frac{1}{2}\right\}$;

(P-3) There exist $\tilde{a}_{n}, \tilde{b}_{n} \in \mathbb{N} \cup\{0\}$ independent of $N$ such that

$$
v_{\infty}\left(Z_{r, 0}^{(n)}(\tau)\right)=\tilde{a}_{n} \text { and } v_{\infty}\left(Z_{r, \frac{1}{2}}^{(n)}(\tau)\right)=\frac{\tilde{b}_{n}}{2}
$$

for any $N \in \mathbb{N}_{\geq 3}$ and $(r, s) \in \mathcal{Q}(N)$ with $s \in\left\{0, \frac{1}{2}\right\}$;

(P-4) $\tilde{a}_{n}=a_{n}$ and $\tilde{b}_{n}=b_{n}$.

Obviously, to prove any one of $(\mathrm{P}-1)-(\mathrm{P}-4)$ is not simple at all. However, it is unexpected that Painlevé VI can be applied to prove those properties.

We would like to study the asymptotics of pre-modular forms $Z_{r, s}^{(n)}(\tau)$ as $\tau \rightarrow \infty$. As discussed earlier, these asymptotics are the most important steps towards the proof of properties (P-2)-(P-4) in Remark 8.5. We only need to consider $\tau \in F_{2}$, where $F_{2}$ is a fundamental domain of $\Gamma(2)$ defined by

$$
F_{2}:=\{\tau \in \mathbb{H}|0 \leq \operatorname{Re} \tau<2,| \tau-1 / 2|\geq 1 / 2,| \tau-3 / 2 \mid>1 / 2\}
$$

The first theorem is concerned with $\operatorname{Re} s \in\left(0, \frac{1}{2}\right)$, which is a stronger version of property (P-2) in Remark 8.5. 
TheOREM 8.6. [9] Given any $n \geq 1$ and $(r, s) \in \mathbb{C}^{2} \backslash \frac{1}{2} \mathbb{Z}^{2}$ such that Re $s \in\left(0, \frac{1}{2}\right)$, there holds

$$
Z_{r, s}^{(n)}(\tau)=\check{Z}^{(n)}(s)+o(1) \text { as } F_{2} \ni \tau \rightarrow \infty,
$$

where $\check{Z}^{(n)}(s)$ is a polynomial of $s$ given as follows:

(1) if $n=2 m+1$ with $m \in \mathbb{N} \cup\{0\}$,

$$
\begin{aligned}
\check{Z}^{(n)}(s)= & (2 \pi)^{2 m(m+1)}[\pi i(2 s-1)]^{m+1} \prod_{k=0}^{m-1}\left[(s+k)\left(s+k+\frac{1}{2}\right)\right. \\
& \left.(s-k-1)\left(s-k-\frac{3}{2}\right)\right]^{m-k} ;
\end{aligned}
$$

(2) if $n=2 m$ with $m \in \mathbb{N}$,

$$
\begin{gathered}
\check{Z}^{(n)}(s)=(-1)^{m^{2}}(2 \pi)^{2 m^{2}}[\pi i(2 s-1)]^{m} s^{m}(s-1)^{m} \prod_{k=0}^{m-2}\left[\left(s+k+\frac{1}{2}\right)\right. \\
\left.(s+k+1)\left(s-k-\frac{3}{2}\right)(s-k-2)\right]^{m-1-k} .
\end{gathered}
$$

In particular,

$$
\lim _{F_{2} \ni \tau \rightarrow \infty} Z_{r, s}^{(n)}(\tau) \neq 0 \text { as long as } \operatorname{Re} s \in(0,1 / 2) \cup(1 / 2,1) .
$$

The final result deals with $s \in\left\{0, \frac{1}{2}\right\}$, which confirms properties (P-3)(P-4) in Remark 8.5. Denote $q=e^{2 \pi i \tau}$. Clearly $q \rightarrow 0$ as $F_{2} \ni \tau \rightarrow \infty$.

TheOREM 8.7. [9] Given any $n \geq 1$ and $(r, s) \in \mathbb{C}^{2} \backslash \frac{1}{2} \mathbb{Z}^{2}$ such that $s \in\left\{0, \frac{1}{2}\right\}$, there hold

$$
\begin{aligned}
& Z_{r, 0}^{(n)}(\tau)=\alpha_{0}^{(n)}(r) q^{a_{n}}+O\left(|q|^{a_{n}+1}\right), \\
& Z_{r, \frac{1}{2}}^{(n)}(\tau)=\beta_{0}^{(n)}(r) q^{\frac{b_{n}}{2}}+O\left(|q|^{\frac{b_{n}+1}{2}}\right),
\end{aligned}
$$

where both $\alpha_{0}^{(n)}(r)$ and $\beta_{0}^{(n)}(r)$ have no rational zeros in $(0,1 / 2) \cup(1 / 2,1)$. Here $\left(a_{n}, b_{n}\right)$ is given by (5.23).

We conclude the introduction by the following remark. To prove Theorem 8.7, it suffices for us to study the asymptotics of $Z_{\frac{1}{4}, 0}^{(n)}(\tau)$. In [9], we could prove

$$
Z_{\frac{1}{4}, 0}^{(n)}(\tau)=(-1)^{C_{n}} 16^{a_{n}} \pi^{\frac{n(n+1)}{2}} \prod_{k=1}^{n-1}(2 k+1)^{n-k} q^{a_{n}}+O\left(|q|^{a_{n}+1}\right),
$$

where

$$
C_{n}=\left\{\begin{array}{l}
\frac{n^{2}-1}{4} \text { if } n \text { is odd } \\
\frac{n^{2}}{4} \text { if } n \text { is even. }
\end{array}\right.
$$


The interesting thing is that every odd positive integer will appear in the coefficient of the leading term $q^{a_{n}}$ as $n$ increases. This phenomena shows that pre-modular form $Z_{r, s}^{(n)}(\tau)$ should possess many interesting unknown properties that are worthy to study in a future work.

\section{References}

[1] L.V. Ahlfors; Complex analysis, An introduction to the theory of analytic functions one complex variable, third edition. McGraw-Hill Higher Education, 1978

[2] M.V. Babich and L.A. Bordag; The elliptic form of the sixth Painlevé equation. Preprint NT Z25/1997, Leipzig (1997).

[3] W. Bergweiler and A. Eremenko; Green's function and anti-holomorphic dynamics on a torus. Proc. Amer. Math. Soc. 144 (2016), no.7, 2911-2922.

[4] F. Beukers and Waall; Lamé equations with algebraic solutions. J. Differ. Equ. 197 (2004), 1-25.

[5] P. Boalch; From Klein to Painlevé via Fourier, Laplace and Jimbo. Proc. Lond. Math. Soc. 90 (2005), 167-208.

[6] C.L. Chai, C.S. Lin and C.L. Wang; Mean field equations, Hyperelliptic curves, and Modular forms: I. Cambridge J. Math. 3 (2015), no. 1-2, 127-274.

[7] Z. Chen, T.J. Kuo and C.S. Lin; Hamiltonian system for the elliptic form of Painlevé VI equation. Preprint 2015. arXiv:1506.06545v1 [math. AG] 2015.

[8] Z. Chen, T.J. Kuo and C.S. Lin; Mean field equation, Isomonodromic deformation and Painlevé VI equation. Preprint.

[9] Z. Chen, T.J. Kuo and C.S. Lin; On the geometry of generalized Lamé equations, in preparation.

[10] Z. Chen, T.J. Kuo, C.S. Lin and C.L. Wang; Green function, Painlevé VI equation, and Eisenstein series of weight one. Preprint 2015. http://www.math.ntu.edu.tw/dragon/works.htm

[11] C.C. Chen and C.S. Lin; Sharp estimates for solutions of multi-bubbles in compact Riemann surfaces. Comm. Pure Appl. Math. 55 (2002), 728-771.

[12] C.C. Chen and C.S. Lin; Topological degree for a mean field equation on Riemann surfaces. Comm. Pure Appl. Math. 56 (2003), 1667-1727.

[13] C.C. Chen and C.S. Lin; Mean field equation of Liouville type with singular data: Topological degree. Comm. Pure Appl. Math. 68 (2015), 887-947.

[14] C.C. Chen, C.S. Lin and G. Wang; Concentration phenomenon of two-vortex solutions in a Chen-Simons model. Ann. Scuola Norm. Sup. Pisa 3 (2004), 367-379.

[15] S. Dahmen; Counting integral Lamé equations with finite monodromy by means of modular forms. Master Thesis, Utrecht University 2003.

[16] S. Dahmen; Counting integral Lamé equations by means of dessins d'enfants. Trans. Amer. Math. Soc. 359 (2007), 909-922.

[17] F. Diamond and J. Shurman; A first course in modular forms. Springer, 2005.

[18] B. Dubrovin and M. Mazzocco; Monodromy of certain Painlevé-VI transcendents and reflection groups. Invent. Math. 141 (2000), 55-147.

[19] R. Fuchs; Über lineare homogene Differentialgleichungen zweiter Ordnung mit drei im Endlichen gelegenen wesentlich singulären Stellen. Math. Ann. 63 (1907), 301-321.

[20] D. Guzzetti; The elliptic representation of the general Painlevé VI equation. Comm. Pure Appl. Math. 55 (2002), 1280-1363.

[21] E. Hecke; Zur Theorie der elliptischen Modulfunctionen. Math. Ann. 97 (1926), 210242.

[22] N.J. Hitchin; Twistor spaces, Einstein metrics and isomonodromic deformations. J. Differ. Geom. 42 (1995), 30-112. 
[23] M. Inaba, K. Iwasaki and M. Saito; Bäcklund transformations of the sixth Painlevé equation in terms of Riemann-Hilbert correspondence. Inter. Math. Res. Not. 1 (2004), $1-30$.

[24] K. Iwasaki, H. Kimura, S. Shimomura and M. Yoshida; From Gauss to Painlevé: A Modern Theory of Special Functions. Aspects of Mathematics, 16, 1991, Springer.

[25] M. Jimbo; Monodromy problem and the boundary condition for some Painlevé equations. Publ. Res. Inst. Math. Sci. 18 (1982), 1137-1161.

[26] R. Lițcanu; Lamé operators with finite monodromy - a combinatorial approach. J. Differ. Equ. 207 (2004), 93-116.

[27] C.S. Lin and C.L. Wang; Mean field equations, Hyperelliptic curves, and Modular forms: II. arXiv:1502.03295v1 [math. AP] 2015.

[28] C.S. Lin and C.L. Wang; Elliptic functions, Green functions and the mean field equations on tori. Annals of Math. 172 (2010), 911-954.

[29] O. Lisovyy and Y. Tykhyy; Algebraic solutions of the sixth Painlevé equation. J. Geom. Phys. 85 (2014), 124-163.

[30] Y. Manin; Sixth Painlevé quation, universal elliptic curve, and mirror of $\mathbb{P}^{2}$. Amer. Math. Soc. Transl. (2), 186 (1998), 131-151.

[31] K. Okamoto; Studies on the Painlevé equations. I. Sixth Painlevé equation $P_{V I}$. Ann. Mat. Pura Appl. 146 (1986), 337-381.

[32] P. Painlevé; Sur les équations différentialles du second ordre à points critiques fixes. C. R. Acad. Sic. Paris Sér. 143 (1906), 1111-1117.

[33] T. Tsudo, K. Okamoto and Sakai; Finding transformations of the Painlevé equations. Math. Ann. 331 (2005), 713-738.

[34] A.V.D. Waall; Lamé Equations with Finite Monodromy. PhD Thesis, Utrecht University, 2002.

[35] E. Whittaker and G. Watson; A course of modern analysis. Cambridge University Press, 1996.

Taida Institute for Mathematical Sciences, National Taiwan University, TAIPEI 10617, TAIWAN

E-mail address: cslin@math.ntu.edu.tw 